\title{
Platelet HMGB1 in Platelet-Rich Plasma Promotes Tendon Wound Healing
}

Jianying Zhang ${ }^{1}$, Feng Li $^{1}$, Tyler Augi ${ }^{1}$, Kelly M. Williamson ${ }^{1}$, Kentaro Onishi ${ }^{1,3}$, MaCalus V. Hogan $^{1,2,3}$, Matthew D. Neal ${ }^{4}$, James H-C. Wang ${ }^{1,2,3 \#}$

${ }^{1}$ MechanoBiology Laboratory, Department of Orthopaedic Surgery,

${ }^{2}$ Department of Bioengineering,

${ }^{3}$ Department of Physical Medicine and Rehabilitation,

${ }^{4}$ Department of Surgery, University of Pittsburgh, Pittsburgh, PA15213

\section{\#Correspondence:}

James H-C. Wang, PhD

MechanoBiology Laboratory

Department of Orthopaedic Surgery

University of Pittsburgh School of Medicine

E1640 BST, 200 Lothrop Street

Pittsburgh, PA15213

Tel. 412-648-9102

Fax: 412-648-8548

Email: wanghc@pitt.edu

Short Title: Platelet HMGB1 Promotes Tendon Healing 


\section{Abstract}

Platelet-rich plasma (PRP) is a widely used autologous treatment for tendon injuries in clinics, but clinical trials often produce conflicting results. Platelets (PLTs) are a major source of high mobility group box1 (HMGB1) that is gaining attention as a chemoattractant that can recruit stem cells to the wound area to enhance healing; however, the contribution of PLT HMGB1 in wounded tendon healing remains unexplored. This study investigated the effect of PLT HMGB1 within PRP to enhance healing in an acute patellar tendon injury model in PLT HMGB1 knockout $(\mathrm{KO})$ mice and GFP mice. A window defect was created in the patellar tendons of both groups of mice, and wounds were treated with either saline, PRP isolated from PLT HMGB1 KO mice, or PRP isolated from GFP mice. Seven days post-treatment, animals were sacrificed and analyzed by gross inspection, histology, and immunostaining for characteristic signs of tendon healing and repair. Our results showed that in comparison to mice treated with PRP from PLT HMGB1-KO mice, wounds treated with PRP from GFP mice healed faster and exhibited a better organization in tendon structure. Mice treated with PRP from PLT HMGB1-KO mice produced tendon tissue with large premature wound areas and low cell densities. However, wounds of PLT HMGB1 KO mice showed better healing with PRP from HMGB1 KO mice compared to saline treatment. Moreover, wounds treated with PRP from GFP mice had increased extracellular HMGB1, decreased CD68, increased stem cell markers CD146 and CD73, and increased collagen III protein expression levels compared to those treated with PRP from PLT HMGB1

67 KO mice. Thus, PLT HMGB1 within PRP plays an important role in the healing of wounded tendon. Our findings also suggest that the efficacy of PRP treatment for tendon injuries in clinics may be affected by PLT HMGB1 within PRP preparations. 
71

72 Tendon injuries to the Achilles and patellar tendons are prevalent in both occupational and

\section{3}

74

\section{Introduction}

athletics populations. Overall, injured tendon healing is slow and yields an inferior quality of

tendon tissue that is prone to re-injury. Many therapeutic approaches including injection of autologous platelet-rich plasma (PRP) have been devised to manage tendon injuries $(1,2)$. The use of PRP is a popular option in the treatment of tendon injuries in orthopaedics and sports medicine $(1,2)$, however the efficacy of PRP treatment on tendon injuries, particularly in clinical trials, has been controversial. Several studies have reported that PRP can effectively treat tendon injuries (3-5), whereas others have shown the opposite with no improvement in pain or tendon function after PRP treatment (6-8). Thus, further investigation into the role of platelets (PLTs) in tendon wound healing is essential to understand the PLT action mechanism in PRP and improve the efficacy of PRP in treating tendon injuries.

Although lacking nuclei, platelets are a rich source of high mobility group box-1 (HMGB1), a highly conserved nuclear protein that is released by all cell types upon injury $(9,10)$. The function of HMGB1 as an inflammatory molecule or chemoattractant is dependent upon its redox state. When inside the cell, either in the nucleus or cytoplasm, HMGB1 is completely reduced as fully reduced HMGB1 (frHMGB1). Once released to the extracellular matrix, frHMGB1 is partially oxidized to disulfide HMGB1 (dsHMGB1) that is believed to initiate inflammation (11). Research has suggested that during platelet activation, HMGB1 is presented on the cell surface and released in significant amounts, with the current theory that HMGB1 is fully reduced at this stage (12-15). Therefore, we hypothesized that platelet HMGB1 (PLT HMGB1) within PRP may have an important role in tendon injury healing. To 
94 test the hypothesis, we investigated the effect of PLT HMGB1 within PRP on wounded tendon

95 healing using a transgenic mouse line with platelet-specific ablation of HMGB1 (PLT

96

97

98

99

100

101

102

103

104

105

106

107

108

109

110

111

112

113

114

115

116

HMGB1-KO). The findings of this study showed that PLT-HMGB1 within PRP preparations is able to facilitate proper healing and repair of tendon injuries.

\section{Materials and methods}

\section{Animals}

All experiments were performed according to the relevant guidelines and regulations. The protocol for animal use was approved by the Institutional Animal Care and Use Committee of the University of Pittsburgh (IACUC protocol \#18083391). C57BL/6-Tg (UBC-GFP) mice were obtained from Jackson Laboratory (Bar Harbor, ME). Mice with platelet-specific ablation of HMGB1 (Pf4-Cre Hmgb1 ${ }^{\mathrm{fl} / \mathrm{fl}}$ mice, referred to as PLT HMGB1-KO mice) were generated as described elsewhere using the Cre/loxP system (16).

\section{Isolation and preparation of platelets and PRP}

Mice were anesthetized with isoflurane, and blood was drawn from the retro-orbital plexus into anti-coagulated tubes. PRP was obtained by centrifugation at $500 \mathrm{~g}$ for $10 \mathrm{~min}$. Platelets were first pelleted from PRP by centrifugation at 1,000g for $10 \mathrm{~min}$, and were then resuspended in ACD buffer consisting of $39 \mathrm{mM}$ citric acid, $75 \mathrm{mM}$ sodium citrate, and $135 \mathrm{mM}$ dextrose with 5 $\mathrm{mM}$ of EDTA according to the published protocol (17). This platelet solution was used for the following experiments. 
117 Isolated platelets were activated by adding $100 \mu \mathrm{l}$ of $10,000 \mathrm{U} / \mathrm{ml}$ bovine thrombin solution into

$1180.4 \mathrm{ml}$ of $1 \times 10^{8} / \mathrm{ml}$ platelet-ACD solution at room temperature for $30 \mathrm{~min}$. The reaction mixture

119 was centrifuged at $1,000 \mathrm{~g}$ for $10 \mathrm{~min}$, and the supernatant was collected to determine the amount

120 of HMGB1 released from platelets using an HMGB1 ELISA kit according to the manufacturer's

121 protocol (Shino-Test Corporation, Tokyo, Japan). The pellet was re-suspended with $0.9 \%$ of

122 sodium chloride solution and reacted with a rabbit anti-mouse HMGB1 primary antibody for 3

123 hrs at room temperature (1:350, abcam, Cat. \#ab18256), followed by a goat anti-rabbit secondary

124 antibody conjugated with Cy3 for $1 \mathrm{hr}$ at room temperature (1:500, Millipore, Cat. \#AP132C).

\section{Mouse tendon wound healing model}

127 The effect of PLT HMGB1 on tendon wound healing was tested with a window defect created in each patellar tendon (PT) of PLT HMGB1-KO mice and GFP mice using a $1 \mathrm{~mm}$ diameter biopsy punch. Wounded PLT HMGB1-KO mice and GFP mice were divided into three groups

(GFP-PRP). All mice were sacrificed at 7 days post-injury, and patellar tendons were harvested.

The effect of PLT HMGB1 on wounded tendon healing and cell migration was assessed by histological analysis.

\section{Histochemical staining on mouse tendon tissue sections}

138 Tendon tissue sections were fixed with 4\% paraformaldehyde for $20 \mathrm{~min}$ at room temperature, 139 and then washed three times with PBS. Slides were stained with H\&E at room temperature 
140 according to the standard protocols, washed with water 3 times, and dehydrated through 15\%,

$14130 \%, 50 \%, 75 \%, 95 \%$ alcohol, and absolute alcohol for five minutes each. Finally, slides were

142 treated with xylene and mounted with resinous mounting medium. The staining results were

143 observed and imaged on a microscope (Nikon eclipse, TE2000-U).

\section{Immunostaining on mouse tendon tissue sections}

146 For immunostaining, the patellar tendons were dissected from the mice and were immediately

147 immersed in O.C.T compound (Sakura Finetek USA Inc., Torrance, CA) in disposable molds

148 and frozen at $-80^{\circ} \mathrm{C}$. Then, cryo-sectioning was performed at $-25^{\circ} \mathrm{C}$ to obtain $\sim 8 \mu \mathrm{m}$ thick tissue

149 sections, which were left at room temperature overnight. The tissue sections were fixed in $4 \%$

150 paraformaldehyde for 15 min and blocked with universal blocking solution (ThermoFisher

151 Scientific, Pittsburgh, PA, Cat. \#37515). The sections were then incubated with rabbit anti-

152 mouse HMGB1 antibody (1:350, abcam, Cat. \#ab18256) at $4^{\circ} \mathrm{C}$ overnight followed by goat anti-

153 rabbit secondary antibody conjugated with $\mathrm{Cy} 3$ for $1 \mathrm{hr}$ at room temperature (1:500, Millipore,

154 Cat. \#AP132C). Since the purpose of this staining was to evaluate the presence of extracellular

155 HMGB1 in the tendon, the tissue sections were not treated with the penetration reagent Triton-

$156 \mathrm{X} 100$ that permeates the nuclear membrane.

158 Similarly, the fixed tissue sections were reacted individually overnight at $4{ }^{\circ} \mathrm{C}$ with the

159 following primary antibodies: rabbit anti-CD68 antibody (1:500, Abcam, Cat. \#125212,

160 Cambridge, MA), rabbit anti-CD146 antibody (1:500, Abcam, Cat. \#75769; Cambridge, MA),

161 rabbit anti-CD73 antibody (1:500, LSBio, Cat. \#LS-B14527-50, Seattle, WA), or rabbit anti- 
162 collagen III antibody (1:500, ThermoFisher, Cat. \#22734-1-AP, Waltham, MA). In the next

163 morning, tissue sections were washed 3 times with PBS and incubated at room temperature for

1642 hrs with Cy3-conjugated goat anti-rabbit IgG antibody (1:500, Millipore, Cat. \#AP132C).

165 Total cell numbers were stained with 4.6-diamidino-2-phenylindole (DAPI). The stained

166 tendon tissue sections were imaged using the fluorescent microscope (Nikon eclipse, TE2000-

$167 \mathrm{U})$.

Semi-quantification of positively stained tissue sections

170 The percentage of HMGB1 expression in activated platelets from PLT HMGB1-KO and GFP

171 mice was determined by semi-quantification. Platelets were stained for HMGB1 (as above) from

172 three mice of each group, smeared onto a glass slide, and imaged using a fluorescent microscope

173 (Nikon eclipse, TE2000-U). The percentages of HMGB1 expression in platelets were calculated

174 by dividing the number of positively stained platelets with red fluorescence by the total number

175 of platelets.

177 For semi-quantification of cell marker staining in tissue sections, stained tissue sections (3

178 sections/mouse) in each group (3 mice/group) were examined under a microscope and five

179 random images were taken. Positively stained areas were manually identified by examining the

180 images taken and processed using SPOT ${ }^{\mathrm{TM}}$ imaging software (Diagnostic Instruments, Inc.,

181 Sterling Heights, MI). The proportion of positive staining was calculated by dividing the total

182 area viewed under the microscope by the positively stained area. These values were averaged to

183 represent the percentage of positive staining in all the groups. 
All results were obtained from 6 tendons (three mice) from each group and presented as the mean \pm SD. The statistical analyses were performed with an unpaired student $t$-test. When $\mathrm{P}<$ 0.05, the two groups in comparison were considered significantly different.

\section{Results}

\section{Expression of HMGB1 is decreased in platelets of PLT HMGB1-KO mice}

First, the expression of PLT HMGB1 was assessed for both transgenic lines, specifically Pf4-Cre Hmgb1 $1^{\mathrm{fl} / \mathrm{fl}}$ mice, referred to as PLT HMGB1-KO mice, and GFP mice. Platelets were isolated and stained as described in the methods. Immunostaining showed that PLT HMGB1 was decreased within PLT HMGB1-KO mice (Fig. 1A, B) compared to GFP mice (Fig. 1C, D). Semi-quantification (Fig. 1E) confirmed these results showing only $\sim 7 \%$ of platelets in PLT HMGB1-KO mice stained positively for HMGB1, but $86 \%$ of platelets in GFP mice stained positively for HMGB1. After verifying both transgenic lines for the level of PLT HMGB1, further experiments were performed using PRP preparations to investigate the role of PLT HMGB1 in healing and repair within PRP preparations.

\section{Fig. 1 KO-PLTs have much less HMGB1 than GFP-PLTs.}

\section{PRP generated from PLT-HMGB1 KO mice impairs tendon wound healing}

Patellar tendon (PT) wounds of mice from each group were treated individually with PRP generated from either PLT HMGB1-KO mice or from GFP mice. A saline treatment was used as a control treatment. Saline treated wound exhibited large unhealed wound areas (red arrows in Fig. 2A, D), whereas wounds treated with either KO or GFP-PRP in both mice groups (Fig. 2B, 
C, E, F) had better healing compared to saline treated wounds. Further gross inspection showed that wound healing in the patellar tendons of PLT HMGB1-KO mice displayed unhealed wound areas in all treatment groups (Fig. 2A-C, yellow and green arrow in $\mathbf{B}$ and $\mathbf{C}$, respectively) compared to GFP mice (Fig. 2E, F). PLT HMGB1-KO mice treated with GFP-PRP (Fig. 2C)

214 exhibited slightly better healing compared to treatment with KO-PRP (Fig. 2B). However, PLT

215 HMGB1-KO mice treated with KO-PRP (Fig. 2B) had better healing than a saline treatment

216 (Fig. 2A, red arrow). These results indicated that PLT HMGB1 within PRP preparations is

217 required for promoting tendon wound healing by PRP.

Fig. 2 PRP generated from PLT HMGB1-KO mice adversely affects wounded patellar

tendon healing. treated mice healed faster (Fig. 3). Specifically, PLT HMGB1-KO mice treated with KO-PRP still exhibited large unhealed areas (black arrows, Fig. 3B) although GFP-PRP treated KO mouse wound displayed better healing (Fig. 3C) compared to that treated with KO-PRP (Fig. 3B). In contrast, GFP-mice treated with GFP-PRP had complete healing with a normal-like tendon appearance (Fig. 3F), while unhealed wound areas were found in GFP mice treated with KO-

229 PRP (Fig. 3E). GFP-PRP treated wounds in the patellar tendon of GFP mice displayed newly 230 formed tendon tissue in the wound area with normal-like tendon organization (Fig. 3F). Saline 231 treated tendons produced large unhealed wound areas in both mice groups (red arrows in Fig. 
233 despite the model or form of PRP used as a treatment (Fig. 3B-F). These results further confirm that PLT HMGB1 within PRP preparations is able to enhance tendon healing, and without PLT HMGB1, tendon wound healing is reduced and slowed in comparison.

241 Previous research has shown that the release of local HMGB1 from injured tissues can enhance

242 the healing and regeneration of skeletal, hematopoietic and muscle tissues in vivo (18). Thus, to

243 further evaluate the role of PLT-HMGB1 in PRP preparations in healing and repair, the patellar

244 tendons from each transgenic injury model with their respective PRP treatments were evaluated

245 for the release of tendon tissue specific HMGB1 at the site of injury. Each patellar tendon was

246 assessed with immunostaining for HMGB1 to determine how the presence or absence of PLT

247 HMGB1 in both the GFP-PRP and KO-PRP may affect healing and repair within our model. Our

248 results showed that the acute injury model induced the release of HMGB1 from local tendon

249 tissue surrounding the wound area to the tendon matrix in both GFP mice and PLT HMGB1-KO

250 mice, as evidenced by positively stained HMGB1 (red fluorescence in Fig. 4). However, reduced

251 levels of HMGB1 can be seen within the tendon matrix of PLT HMGB1-KO mice (Fig. 4A-L)

252 in comparison to the elevated level of HMGB1 released in tendons of GFP mice (Fig. 4M-X).

253 Fluorescent image analysis indicated that GFP-PRP treatment increased the levels of HMGB1 in

254 the tendon matrix of both transgenic mouse lines specifically due to treatment with GFP-PRP

255 (Fig. 4I-L, 4U-X). However, the concentration of locally released HMGB1 in the tendons treated 
with PLT HMGB1-KO-PRP were not significantly increased (Fig. 4E-H). Thus, PLT HMGB1 is able to enhance the presence of local HMGB1 at the injury site.

Fig. 4 HMGB1 expression in KO-PRP treated wound is much lower than GFP-PRP treated wound.

\section{PRP from PLT-HMGB1-KO mice increases inflammation in wounded tendon}

Collected patellar tendon tissues were analyzed for CD68, a marker of M1 pro-inflammatory macrophages $(19,20)$, using immunostaining. Overall, the levels of CD68 in the wound areas of CD68 positive cells were found in wounded tendons of both groups of mice treated with saline

267 (Fig. 5A, B, 5G, H) suggesting a high level of tissue inflammation. In PLT HMGB1-KO mice, GFP-PRP treatment decreased CD68 expression (Fig. 5E, F) compared to PRP from KO mice

(Fig. 5C, D). However, in GFP mice, treatment with GFP-PRP significantly decreased positively stained CD68 cells (Fig. 5K, L) compared to the same group treated with PRP from KO mice

(Fig. 5I, J). Taken together, these results suggest that ablation of PLT HMGB1 in PRP results in

272 significant levels of $\mathrm{CD} 68^{+} \mathrm{M} 1$ macrophages in treated tendon tissues, while M1 cells are greatly

273 reduced in tendons treated with normal PRP treatments. Semi-quantification supports these

274 results (Fig. 5M), showing that GFP-PRP is able to reduce the level of CD68 $8^{+}$1 macrophages

275 in both transgenic mouse lines, while both saline and KO-PRP are largely similar in the level of $276 \mathrm{CD}^{2} 8^{+}$cells. 
Fig. 5 Macrophage marker CD68 expression is much higher in KO-PRP treated wound than GFP-PRP treated wound.

Stem cell marker expression is reduced in wounded mouse tendons treated with PRP from

HMGB1 have been shown to enhance tissue repair by recruiting resident stem cells (21). Thus, immunostained for CD146 and CD73 stem cell marker expression $(22,23)$. GFP-PRP treatment of both transgenic mouse lines recruited stem cells to the wound areas, as shown by elevated CD146 ${ }^{+}$cells within GFP-PRP treated tendons $(\mathbf{F i g} . \mathbf{6 E}, \mathbf{F}, \mathbf{6 K}, \mathbf{L})$ compared to tendons treated with KO-PRP (Fig. 6C, D, 6I, J). However, few CD146 ${ }^{+}$cells can be seen in the saline-treated tendons of PLT-HMGB1-KO mice (Fig. 6A, B) compared to saline treatment of GFP mice (Fig. 6G, H). Overall, higher levels of CD146 ${ }^{+}$cells were found in the tendons of GFP mice (Fig. 6GL) compared to PLT HMGB1-KO tendons (Fig. 6A-F). Semi-quantification supports these results (Fig. 6M), showing that GFP-PRP treatment elevated CD146 ${ }^{+}$cells in both transgenic

293 lines, with the highest levels found within GFP mice. 
treated GFP mice (Fig. 7G, H). Overall, both PLT HMGB1-KO PRP and GFP-PRP were able to increase the level of $\mathrm{CD}^{+} 3^{+}$cells but to different degrees. GFP-PRP treatment increased CD73 cells in both mouse lines (Fig. 7E, F, 7K, L), surpassing the effect of PLT HMGB1-KO PRP on CD73 levels (Fig. 7C, D, 7I, J). Semi-quantification of CD73 staining supports these results (Fig. 7M), with GFP-PRP treatment producing elevated $\mathrm{CD} 73^{+}$cells in both transgenic lines with the highest levels in the treated tendons of GFP mice. Taken together, these results suggest that HMGB1 ablation in PLTs can negatively affect tendon wound healing by decreasing stem cell recruitment. compared to GFP-PRP treated wound.

313 Collagen type III (Col III) has an important role in the healing process of tendon (24).

314 Immunostaining for Col III was carried out to assess healing within wounded and treated patellar 315 tendons. Overall, Col III expression was higher in the treated tendons of GFP mice (Fig. 8G-L) compared to tendons in PLT HMGB1-KO mice (Fig. 8A-F). Also, GFP-PRP treatment increased

317 Col III levels in wounded tendons in both mouse lines (Fig. 8E, F, 8K, L), compared to PLT 318 HMGB1-KO PRP treatment (Fig. 8C, D, 8I, J). The results indicate that in contrast to normal 319 PLTs in GFP mice, HMGB1-ablated PLTs impair tendon wound healing due to decreased Col III 320 levels. Semi-quantification supports this conclusion (Fig. 8M), with the GFP-PRP treatment 321 surpassing the KO-PRP treatment in Col III production. Finally, similar to other results above, 322 GFP-PRP treatment was able to produce the highest Col III production in GFP mice. 


\section{Discussion}

This study investigated the effect of platelet-derived HMGB1 in PRP on wounded tendon healing using a transgenic mouse line with a specific platelet HMGB1 ablation. Our results have demonstrated that in an acute patellar tendon injury model treated with PRP from mice lacking platelet-derived HMGB1, tendon healing was impaired. Impaired healing was characterized by a decrease in local HMGB1 release from injured tissues, an increase in CD68 ${ }^{+} \mathrm{M} 1$ macrophage cells, a decrease in $\mathrm{CD} 146^{+}$and $\mathrm{CD} 3^{+}$stem cells, and a decrease in Col III content. In contrast, treatment with PRP from GFP-mice increased local HMGB1 concentrations in the wound area, reduced the recruitment of inflammatory cells to wounded tendons, increased the recruitment of stem cells, and increased Col III levels. Although the wounds treated with PLT HMGB1-KO-

337 PRP healed faster than the wounds treated with saline, they healed much slower in comparison to 338 wounds treated with GFP-PRP. This slowed healing is likely due to the ablation of PLT HMGB1 339 in these mice, with our results showing the expression of minimal amounts of PLT HMGB1

340 (Fig. 1) that may still facilitate some level of healing compared to the saline treatment. Our 341 results also demonstrated that GFP-PRP treatment enhanced wounded tendon healing in both 342 mouse lines, however to a greater extent in GFP mice than in PLT HMGB1-KO mice. In GFP 343 mice, endogenous HMGB1 in addition to PLT HMGB1 within GFP-PRP were collectively 344 involved in enhancing tendon healing. Thus, our results showed that HMGB1 in platelets is a 
critical factor in PRP treatment, and the efficacy of PRP treatment for tendon injuries in clinics may depend on the level of PLT HMGB1 within PRP preparations.

Tendon injury is one of the most common musculoskeletal injuries that can persist for years with poorly repaired tendon leading to further re-injury. In current clinical practices, PRP is widely used to treat tendon injuries $(2,25)$, with a number of studies showing that PRP treatment promotes the healing and physical function of wounded tendon $(1,2,5,26)$. These beneficial effects are attributed to the platelets in PRP, the role of which is well-established in wound healing and tissue repair (27). In fact, platelets are the "first responders" during wounding that trigger platelet activation and aggregation (28). Once activated, platelets release many factors (e.g. cytokine and growth factors) to enhance healing of injured tissues (29).

In particular, HMGB1, which is abundant in platelets $(13,15,30)$, is also released by activated platelets. It has been shown that HMGB1 released by injured tissues promotes tissue repair by inducing migration and proliferation of stem cells (31-33). Locally released HMGB1 recruits bone marrow-derived mesenchymal stem cells (MSCs), and promotes the proliferation and differentiation of tissue-associated resident stem cells $(11,33,34)$. For instance, vascularization of regenerating tissue is compromised in the absence of leukocyte HMGB1 in a mouse model lacking HMGB1 in the hematopoietic system, but vessel number and vascular area were significantly higher in the presence of leucocyte HMGB1 (35). This study indicated that leukocyte HMGB1 controls the nutrient and oxygen supply to the regenerating tissue. A study demonstrating the role of HMGB1 in muscle regeneration has shown that heterozygous $\mathrm{HMGB}^{+/-}$mice, which express 50\% less HMGB1 when compared with wild type mice, have 
delayed muscle regeneration after acute injury (36). Fully reduced frHMGB1 has been gaining much attention as a chemoattractant that orchestrates tissue regeneration $(18,21)$. This subsequent increase in stem cells in response to HMGB1 administration suggested that the regenerative properties of HMGB1 were mediated by muscle stem cells and high expression of HMGB1 is required for optimal skeletal muscle regeneration (21). Exogenous administration of a single dose of HMGB1, either locally or systemically, promoted tissue repair by targeting endogenous stem cells. Using $\mathrm{HMGB}^{-/-}$mice, they identified the underlying mechanism as the transition of quiescent stem cells from $G_{0}$ to $G_{\text {Alert }}$, and these primed cells rapidly respond to appropriate activating factors released upon injury. These studies support our findings by highlighting the role of HMGB1 as a chemoattractant that promotes regeneration of wounded tendons by recruiting stem cells to injury site.

Macrophages are known to play an essential role in orchestrating inflammation and tissue repair (37). Macrophages secrete various growth factors and signaling molecules and are thus involved in the regulation of inflammation, wound healing, and tissue repair (38). Our findings show that active inflammation is present within wounds treated with PRP from PLT HMGB1-KO mice as indicated by the presence of $\mathrm{CD} 68^{+} \mathrm{M} 1$ macrophages (39), in contrast those treated with PRP from GFP mice. Inflammation can have a detrimental effect on healing. Chronic wounds fail to heal because they are stalled in an early inflammatory state during wound healing (40). For acute injuries, prolonged inflammation can lead to slow healing which may cause the wound to enter a chronic state and fail to heal (41). Thus, controlling inflammation may be an important step in preventing certain acute injuries from progressing to a chronic state. Research has shown that HMGB1 is able to mediate macrophage polarization (42). M1 macrophages are characterized by 
a proinflammatory phenotype which produces proinflammatory cytokines, phagocytosis of microbes, and initiate an immune response (19). M2 phenotype macrophages are a tissue-healing phenotype that releases more HMGB1, which may activate stem cells and promote tissue healing

$394(43,44)$. The switch from a proinflammatory M1 to a tissue-healing M2 phenotype in macrophages is an essential step in muscle regeneration to limit the inflammatory response (20, 45). Our results have shown that local HMGB1 is released in high levels in the wounded tendon matrix when treated with PRP from GFP mice as opposed to low levels of local HMGB1 release due to treatment with KO-PRP. This elevated level of local HMGB1 expression may help switch research however is needed to evaluate the effect of PLT HMGB1 on the M2 phenotype and on macrophage polarization in an acute tendon injury model.

As described above, many studies have demonstrated that HMGB1 activates endogenous stem cells to accelerate tissue regeneration $(18,21)$. Our results indicated that tendon wounds treated with PRP from mice lacking platelet-derived HMGB1 harbor a reduced number of CD146 ${ }^{+}$and

$406 \mathrm{CD}^{+}{ }^{+}$stem cells (Fig. 6, 7). Our results indicate that both platelet and local HMGB1 facilitates

407 stem cell migration in normal wound healing. Our results also demonstrate high level of Col III 408 expression in wounds treated by normal PRP suggesting normal wound healing is occurring, in 409 contrast to the low level in KO mice PRP treated ones. Although Col III is not a major 410 component of the normal tendon, it is believed to play an important role during the healing

411 process because of its ability to form rapid crosslinks and stabilize the repair site (24). 
413 Certain limitations are present within our study. Primarily, our resource for PLT HMGB1-KO

414 mice is limited and as such our study only evaluated healing and repair at a single, relatively

415 short timepoint (or 7 days post-injury) and only focused on the assessment of a limited number

416 of cell markers. Further research is needed within a longer healing timeframe to further assess

417 the effect of the ablation of PLT HMGB1 on tendon healing and repair.

420 in healing wounded tendon by decreasing inflammation, increasing local HMGB1 levels, and

421 recruiting stem cells to the wound area. These results provide the first evidence for the role of

422 HMGB1 within PRP as a therapeutic treatment to promote tendon wound healing. Our findings

423 suggest that the efficacy of PRP treatment for tendon injuries in clinics may depend on PLT

424 HMGB1 within PRP preparations.

\section{Acknowledgements}

427 We thank Dr. Bhavani P Thampatty for assistance in the preparation of this manuscript.

Fig. 1 KO-PLTs have much less HMGB1 than GFP-PLTs. A, B: HMGB1 expression in the

432 platelets of HMGB1-KO mice (KO-PLT); C, D: HMGB1 expression in the platelets of GFP

433 mice (GFP-PLT); B, D: Enlarged images of the box areas in the image A and C. E: Semi-

434 quantification of positively stained platelets with HMGB1 confirms the results of 
435 immunostaining showing that only a few ( 7\%) platelets in HMGB1-KO mice are positively

436 stained with HMGB1 compared to more than $86.8 \%$ of platelets in GFP mice were positively

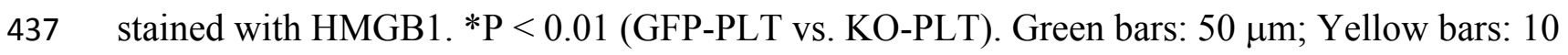

$438 \mu \mathrm{m}$.

Fig. 2 PRP generated from PLT HMGB1-KO mice adversely affects wounded PT healing.

Results show that wounded PTs of PLT HMGB1-KO mice (KO mice) healed slower (A-C, red,

442 yellow, and green arrows point to unhealed area) than PTs in GFP mice (D-F). However, PRP

443

(KO-PRP or GFP-PRP) treated wound $(\mathbf{B}, \mathbf{C}, \mathbf{E}, \mathbf{F})$ healed much faster than the wounds treated

with saline (A, D, red arrows). PT: patellar tendon; KO-PRP: PRP prepared from PLT HMGB1-

KO mice; and GFP-PRP: PRP prepared from GFP mice.

Fig. 3 Wounded PTs treated with KO-PRP heals much slower than those treated with

GFP-PRP. Results show that wounded patellar tendons in PLT HMGB1-KO mice heal slower

449 (A-C, black arrows in $\mathbf{B}$ and $\mathbf{C}$ show unhealed and disorganized area) than GFP mice (D, F).

PRP treated wounds $(\mathbf{B}, \mathbf{C}, \mathbf{E}, \mathbf{F})$ healed much faster than wounded patellar tendons treated with 
$457 \mathbf{X})$ have much more HMGB1 than the wounds treated with saline (A-D, and M-P). KO-PRP: PRP from PLT HMGB1-KO mice; and GFP-PRP: PRP from GFP mice. White bars: $200 \mu \mathrm{m}$, Yellow bars: $50 \mu \mathrm{m}$ (immunostaining).

Fig. 5 Macrophage marker CD68 expression is much higher in KO-PRP treated wound HMGB1-KO mouse patellar tendons (A-F) than that of GFP mouse tendons (G-L). GFP-PRP treated wounds $(\mathbf{A}, \mathbf{B}$, and $\mathbf{G}, \mathbf{H})$. Semi-quantification $(\mathbf{M})$ confirms the results. ${ }^{*} \mathrm{P}<0.01(\mathrm{KO}$ PRP vs. GFP-PRP). KO-PRP: PRP from PLT HMGB1-KO mice; and GFP-PRP: PRP from GFP mice. White bars: $200 \mu \mathrm{m}$; Yellow bars: $50 \mu \mathrm{m}$ (immunostaining). treated mice (A-F) compared GFP tendons which have much higher level of CD146 expression

472 (G-L). GFP-PRP treated wound areas (E, F and K, L) have much more CD146 than the wounds 473 treated with saline (A-D and G-J). Semi-quantification $(\mathbf{M})$ confirms the results. $*$ P $<0.01($ KOPRP vs. GFP-PRP). KO-PRP: PRP from PLT HMGB1-KO mice; and GFP-PRP: PRP from GFP mice. White bars: $200 \mu \mathrm{m}$; Yellow bars: $50 \mu \mathrm{m}$ (immunostaining). 
of KO-PRP mouse patellar tendons (A-F) compared to GFP tendons, which have much higher levels of CD73 expression(G-L). However, both KO-PRP treated and GFP-PRP treated wound areas (C-F, and I-L) have more CD73 than the wounds treated with saline (A, B, and $\mathbf{G}, \mathbf{H})$. Semi-quantification $(\mathbf{M})$ confirms the results. ${ }^{*} \mathrm{P}<0.01$ (KO-PRP vs. GFP-PRP). KO-PRP: PRP from PLT HMGB1-KO mice; GFP-PRP: PRP from GFP mice. White bars: $200 \mu \mathrm{m}$; Yellow bars: $50 \mu \mathrm{m}$ (immunostaining).

\section{Fig. 8 Tendon matrix marker collagen III expression is much lower in KO-PRP treated}

wound than GFP-PRP treated wound. Very low levels of collagen III are found in the wound areas of KO-PRP mouse patellar tendons (A-F) compared to GFP tendons with higher levels of Col III (G-L). KO-PRP and GFP-PRP treated wound areas (C-F, and I-L) have more Col III than the wounds treated with saline $(\mathbf{A}, \mathbf{B}$, and $\mathbf{G}, \mathbf{H})$. Semi-quantification $(\mathbf{M})$ confirms the results. ${ }^{*} \mathrm{P}<0.01$ when $\mathrm{KO}-\mathrm{PRP}$ treatment is compared to GFP-PRP treatment. KO-PRP: PRP from PLT HMGB1-KO mice; GFP-PRP: PRP from GFP mice. Col III: Collagen type III. White bars: $200 \mu \mathrm{m}$; Yellow bars: $50 \mu \mathrm{m}$ (immunostaining).

\section{References}

1. Andia I, Sanchez M, Maffulli N. Tendon healing and platelet-rich plasma therapies. Expert Opin Biol Ther. 2010;10(10):1415-26.

2. Andia I, Martin JI, Maffulli N. Advances with platelet rich plasma therapies for tendon regeneration. Expert Opin Biol Ther. 2018:1-10.

3. Kon E, Filardo G, Di Martino A, Marcacci M. Platelet-rich plasma (PRP) to treat sports injuries: evidence to support its use. Knee Surg Sports Traumatol Arthrosc. 2011;19(4):516-27. 
4. Monto RR. Platelet rich plasma treatment for chronic Achilles tendinosis. Foot Ankle Int. 2012;33(5):379-85.

5. Dragoo JL, Wasterlain AS, Braun HJ, Nead KT. Platelet-rich plasma as a treatment for patellar tendinopathy: a double-blind, randomized controlled trial. Am J Sports Med. 2014;42(3):610-8.

6. de Vos RJ, Weir A, van Schie HT, Bierma-Zeinstra SM, Verhaar JA, Weinans H, et al. Platelet-rich plasma injection for chronic Achilles tendinopathy: a randomized controlled trial. JAMA : the journal of the American Medical Association. 2010;303(2):144-9.

7. de Jonge S, de Vos RJ, Weir A, van Schie HT, Bierma-Zeinstra SM, Verhaar JA, et al. One-year follow-up of platelet-rich plasma treatment in chronic Achilles tendinopathy: a doubleblind randomized placebo-controlled trial. Am J Sports Med. 2011;39(8):1623-9.

8. Schepull T, Kvist J, Norrman H, Trinks M, Berlin G, Aspenberg P. Autologous platelets have no effect on the healing of human achilles tendon ruptures: a randomized single-blind study. Am J Sports Med. 2011;39(1):38-47.

9. Scaffidi P, Misteli T, Bianchi ME. Release of chromatin protein HMGB1 by necrotic cells triggers inflammation. Nature. 2002;418(6894):191-5.

10. Andersson U, Rauvala H. Introduction: HMGB1 in inflammation and innate immunity. J Intern Med. 2011;270(4):296-300.

11. Venereau E, Ceriotti C, Bianchi ME. DAMPs from Cell Death to New Life. Frontiers in immunology. 2015;6:422.

12. Maugeri N, Franchini S, Campana L, Baldini M, Ramirez GA, Sabbadini MG, et al. Circulating platelets as a source of the damage-associated molecular pattern HMGB1 in patients with systemic sclerosis. Autoimmunity. 2012;45(8):584-7.

13. Rouhiainen A, Imai S, Rauvala H, Parkkinen J. Occurrence of amphoterin (HMG1) as an endogenous protein of human platelets that is exported to the cell surface upon platelet activation. Thromb Haemost. 2000;84(6):1087-94.

14. Vogel S, Chatterjee M, Metzger K, Borst O, Geisler T, Seizer P, et al. Activated platelets interfere with recruitment of mesenchymal stem cells to apoptotic cardiac cells via high mobility group box 1/Toll-like receptor 4-mediated down-regulation of hepatocyte growth factor receptor MET. J Biol Chem. 2014;289(16):11068-82.

15. Stark K, Philippi V, Stockhausen S, Busse J, Antonelli A, Miller M, et al. Disulfide HMGB1 derived from platelets coordinates venous thrombosis in mice. Blood. 2016;128(20):2435.

16. Vogel S, Bodenstein R, Chen Q, Feil S, Feil R, Rheinlaender J, et al. Platelet-derived HMGB1 is a critical mediator of thrombosis. The Journal of clinical investigation. 2015;125(12):4638-54.

17. Mardente S, Mari E, Massimi I, Tafani M, Guerriero R, Morsilli O, et al. From Human Megakaryocytes to Platelets: Effects of Aspirin on High-Mobility Group Box 1/Receptor for Advanced Glycation End Products Axis. Frontiers in immunology. 2017;8:1946.

18. Lee G, Espirito Santo AI, Zwingenberger S, Cai L, Vogl T, Feldmann M, et al. Fully reduced HMGB1 accelerates the regeneration of multiple tissues by transitioning stem cells to GAlert. Proceedings of the National Academy of Sciences of the United States of America. 2018;115(19):E4463-E72.

19. Barros MH, Hauck F, Dreyer JH, Kempkes B, Niedobitek G. Macrophage polarisation: an immunohistochemical approach for identifying M1 and M2 macrophages. PLoS One. 2013;8(11):e80908. 
20. Wang H, Melton DW, Porter L, Sarwar ZU, McManus LM, Shireman PK. Altered macrophage phenotype transition impairs skeletal muscle regeneration. Am J Pathol. 2014;184(4):1167-84.

21. Tirone M, Tran NL, Ceriotti C, Gorzanelli A, Canepari M, Bottinelli R, et al. High mobility group box 1 orchestrates tissue regeneration via CXCR4. The Journal of experimental medicine. 2018;215(1):303-18.

22. Dominici M, Le Blanc K, Mueller I, Slaper-Cortenbach I, Marini F, Krause D, et al. Minimal criteria for defining multipotent mesenchymal stromal cells. The International Society for Cellular Therapy position statement. Cytotherapy. 2006;8(4):315-7.

23. Lv FJ, Tuan RS, Cheung KM, Leung VY. Concise review: the surface markers and identity of human mesenchymal stem cells. Stem cells (Dayton, Ohio). 2014;32(6):1408-19. 24. Liu SH, Yang RS, al-Shaikh R, Lane JM. Collagen in tendon, ligament, and bone healing. A current review. Clin Orthop Relat Res. 1995(318):265-78.

25. Foster TE, Puskas BL, Mandelbaum BR, Gerhardt MB, Rodeo SA. Platelet-rich plasma: from basic science to clinical applications. Am J Sports Med. 2009;37(11):2259-72.

26. Kon E, Filardo G, Delcogliano M, Presti ML, Russo A, Bondi A, et al. Platelet-rich plasma: new clinical application: a pilot study for treatment of jumper's knee. Injury. 2009;40(6):598-603.

27. Menter DG, Kopetz S, Hawk E, Sood AK, Loree JM, Gresele P, et al. Platelet "first responders" in wound response, cancer, and metastasis. Cancer Metastasis Rev. 2017;36(2):199213.

28. Menter DG, Tucker SC, Kopetz S, Sood AK, Crissman JD, Honn KV. Platelets and cancer: a casual or causal relationship: revisited. Cancer Metastasis Rev. 2014;33(1):231-69. 29. Eisinger F, Patzelt J, Langer HF. The Platelet Response to Tissue Injury. Front Med (Lausanne). 2018;5:317.

30. Maugeri N, Campana L, Gavina M, Covino C, De Metrio M, Panciroli C, et al. Activated platelets present high mobility group box 1 to neutrophils, inducing autophagy and promoting the extrusion of neutrophil extracellular traps. Journal of Thrombosis and Haemostasis. 2014;12(12):2074-88.

31. Palumbo R, Bianchi ME. High mobility group box 1 protein, a cue for stem cell recruitment. Biochemical pharmacology. 2004;68(6):1165-70.

32. Chavakis E, Hain A, Vinci M, Carmona G, Bianchi ME, Vajkoczy P, et al. High-mobility group box 1 activates integrin-dependent homing of endothelial progenitor cells. Circulation research. 2007;100(2):204-12.

33. Tamai K, Yamazaki T, Chino T, Ishii M, Otsuru S, Kikuchi Y, et al. PDGFRalphapositive cells in bone marrow are mobilized by high mobility group box 1 (HMGB1) to regenerate injured epithelia. Proceedings of the National Academy of Sciences of the United States of America. 2011;108(16):6609-14.

34. Zhang X, Jiang H, Gong Q, Fan C, Huang Y, Ling J. Expression of high mobility group box 1 in inflamed dental pulp and its chemotactic effect on dental pulp cells. Biochem Biophys Res Commun. 2014;450(4):1547-52.

35. Campana L, Santarella F, Esposito A, Maugeri N, Rigamonti E, Monno A, et al. Leukocyte HMGB1 is required for vessel remodeling in regenerating muscles. J Immunol. 2014;192(11):5257-64. 
593 36. Dormoy-Raclet V, Cammas A, Celona B, Lian XJ, van der Giessen K, Zivojnovic M, et 594 al. HuR and miR-1192 regulate myogenesis by modulating the translation of HMGB1 mRNA. $595 \quad$ Nat Commun. 2013;4:2388.

596 37. Oishi Y, Manabe I. Macrophages in inflammation, repair and regeneration. Int Immunol. 597 2018;30(11):511-28.

598 38. Baum CL, Arpey CJ. Normal cutaneous wound healing: clinical correlation with cellular 599 and molecular events. Dermatol Surg. 2005;31(6):674-86; discussion 86.

600 39. Holness CL, Simmons DL. Molecular cloning of CD68, a human macrophage marker 601 related to lysosomal glycoproteins. Blood. 1993;81(6):1607-13.

602 40. Zhao R, Liang H, Clarke E, Jackson C, Xue M. Inflammation in Chronic Wounds.

603 International journal of molecular sciences. 2016;17(12).

604 41. Serra MB, Barroso WA, da Silva NN, Silva SDN, Borges ACR, Abreu IC, et al. From 605 Inflammation to Current and Alternative Therapies Involved in Wound Healing. International 606 journal of inflammation. 2017;2017:3406215.

607 42. Son M, Porat A, He M, Suurmond J, Santiago-Schwarz F, Andersson U, et al. C1q and 608 HMGB1 reciprocally regulate human macrophage polarization. Blood. 2016;128(18):2218-28.

609 43. Bianchi ME, Crippa MP, Manfredi AA, Mezzapelle R, Rovere Querini P, Venereau E. 610 High-mobility group box 1 protein orchestrates responses to tissue damage via inflammation, 611 innate and adaptive immunity, and tissue repair. Immunol Rev. 2017;280(1):74-82.

612 44. Wynn TA, Vannella KM. Macrophages in Tissue Repair, Regeneration, and Fibrosis. 613 Immunity. 2016;44(3):450-62.

614 45. Saclier M, Cuvellier S, Magnan M, Mounier R, Chazaud B. Monocyte/macrophage 615 interactions with myogenic precursor cells during skeletal muscle regeneration. FEBS J.

$616 \quad 2013 ; 280(17): 4118-30$.

617 46. Roszer T. Understanding the Mysterious M2 Macrophage through Activation Markers 618 and Effector Mechanisms. Mediators Inflamm. 2015;2015:816460. 


\section{KO-PLT}

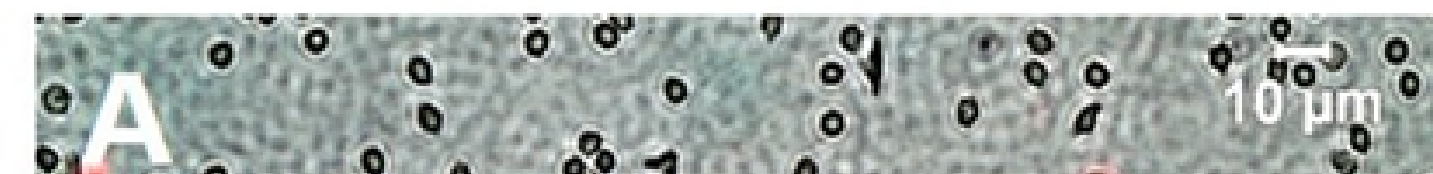
$0.0 \% 1 \%$ ? $\because \because 8 \% 0 \%$ $\because 0 \% 000^{\circ}: 1^{0} 00_{0}^{0}$ $0 \quad 0_{0.800000}^{0}$ $\because 00000: 0 \% 0 \%$

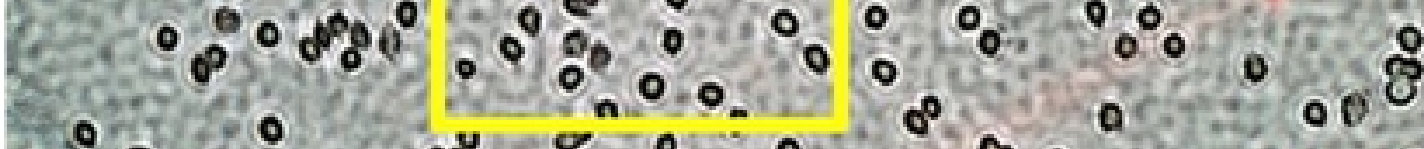

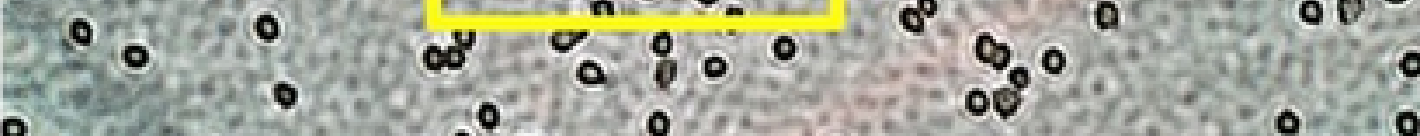

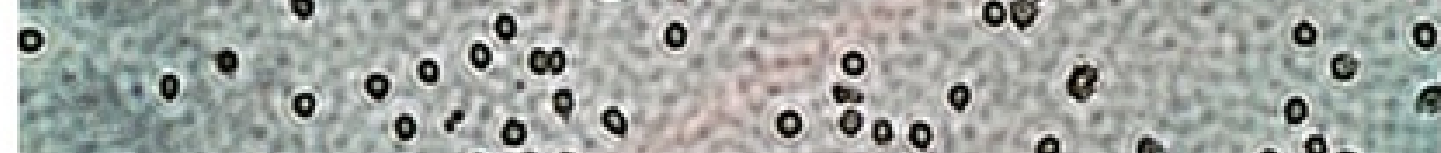
$0 \quad 0.00 \quad 00 \%$ $80000 \% 0 \%$ $\therefore 00^{\circ} \quad 08^{\circ} \quad 00^{\circ} 0^{\circ}$ $\because 0 \% \quad \because 0 \%$ \& $011^{0}$

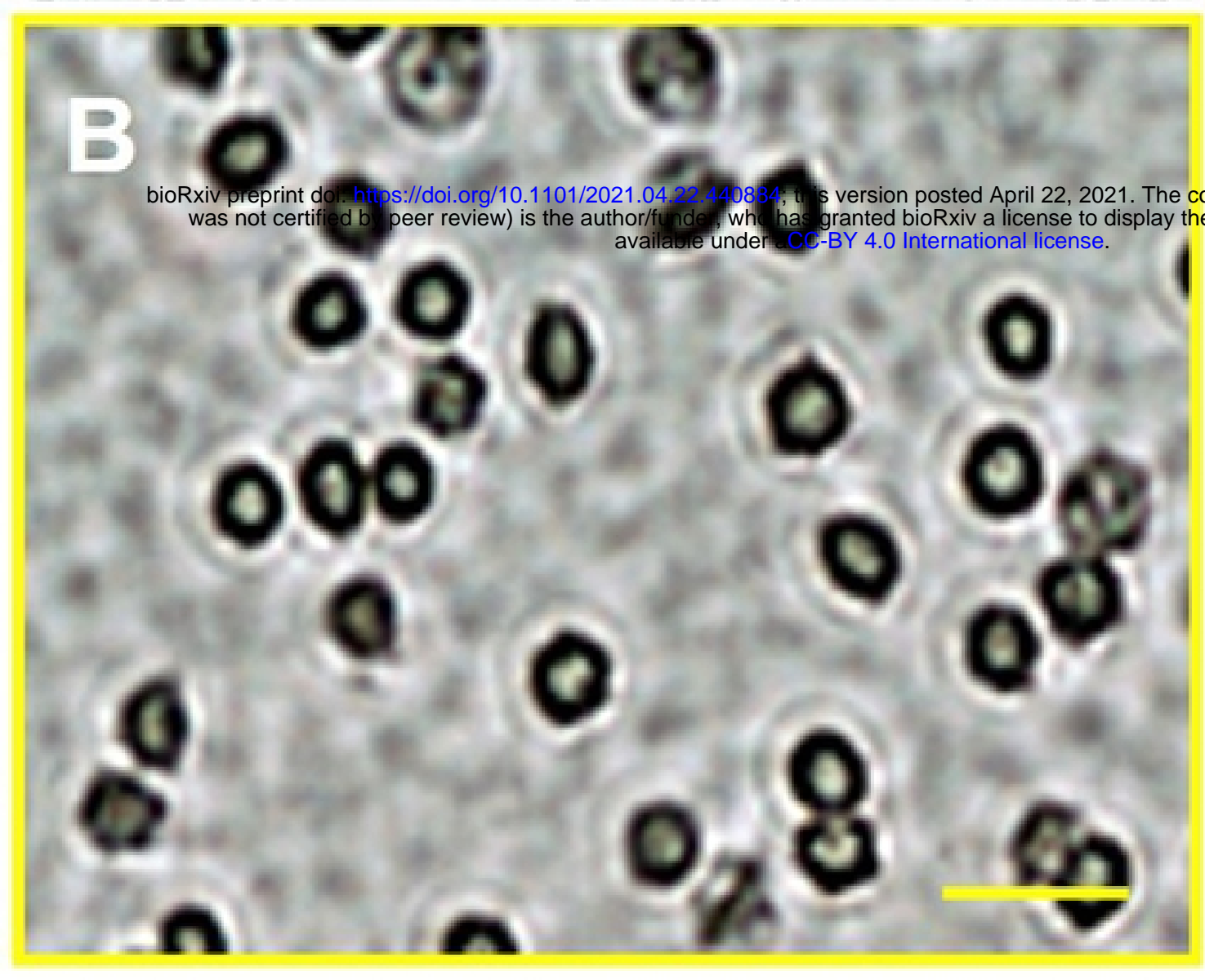

\section{GFP-PLT}
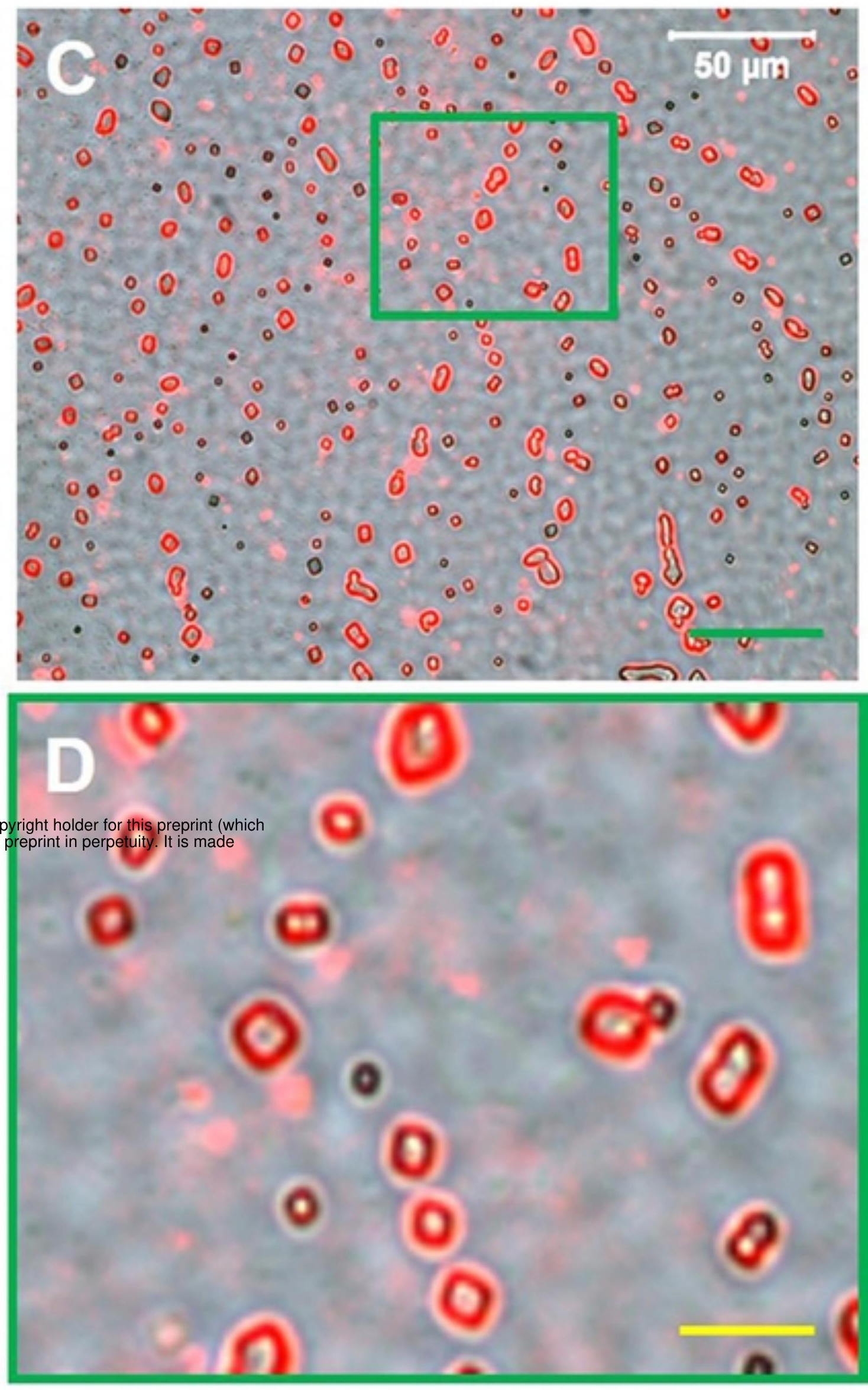

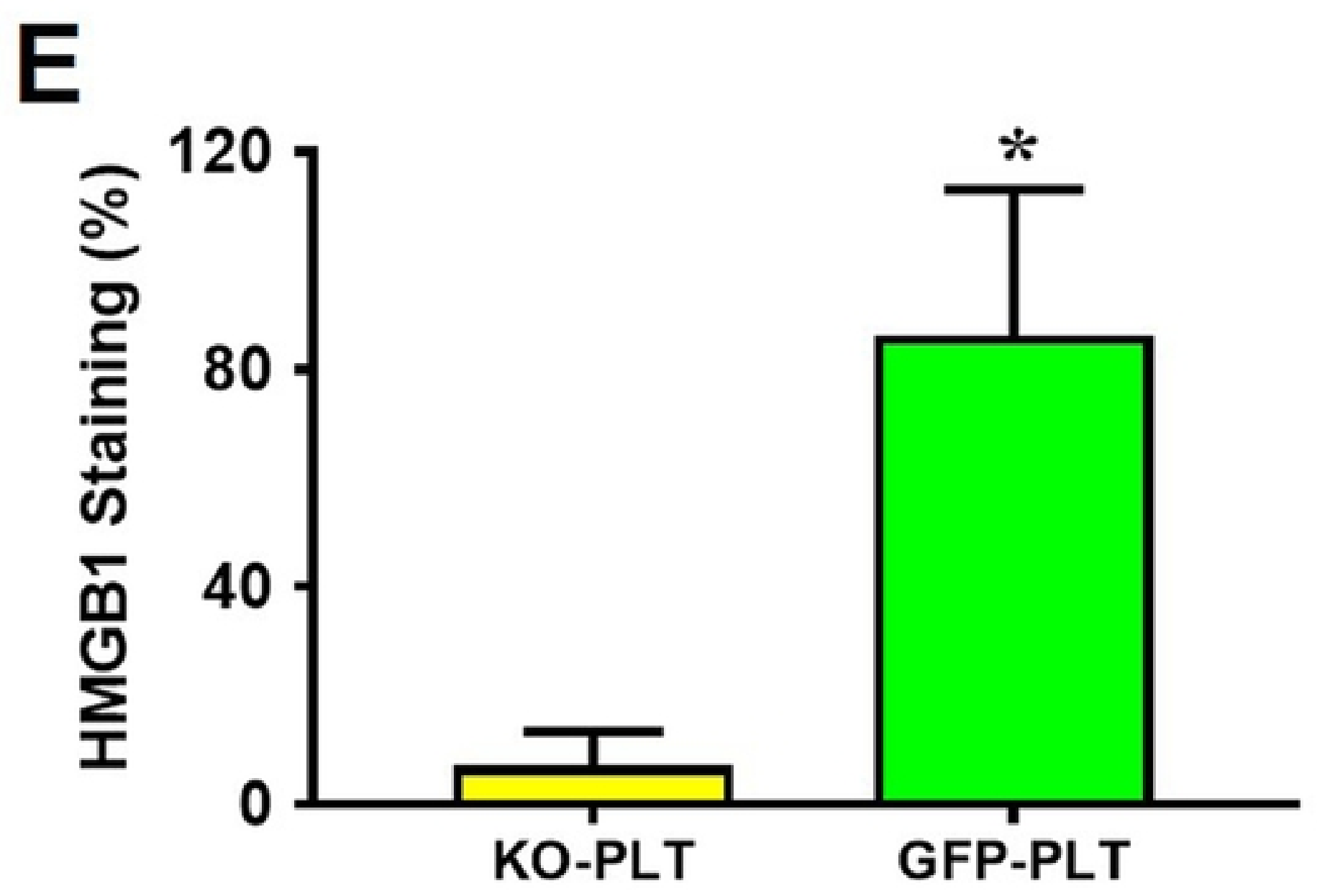

Fig1 


\section{KO mice}

\section{GFP mice}

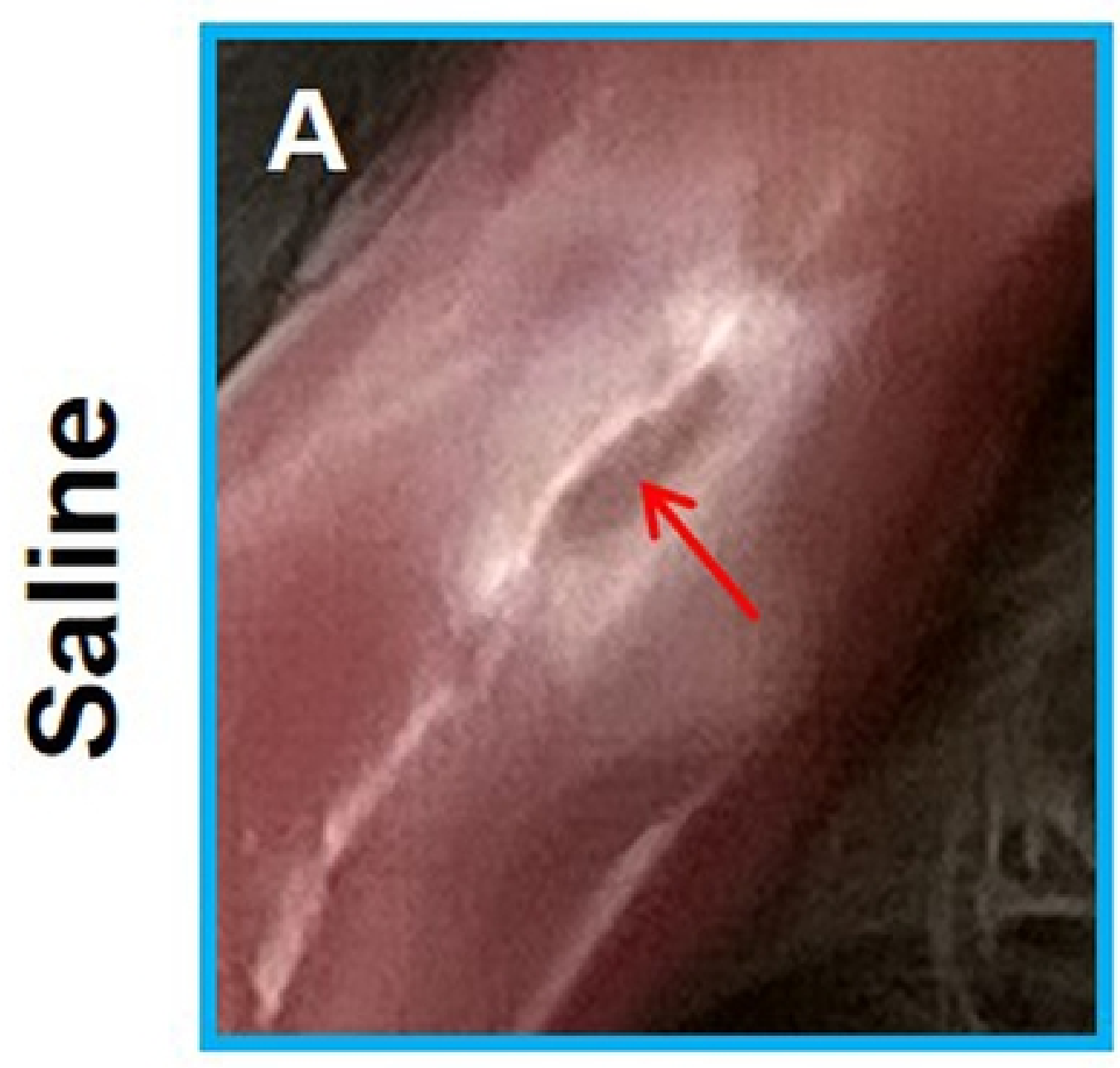

D

B

E

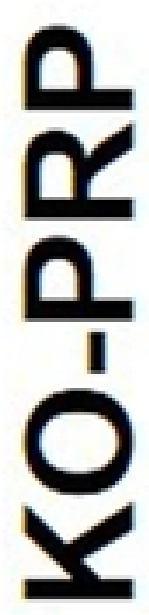
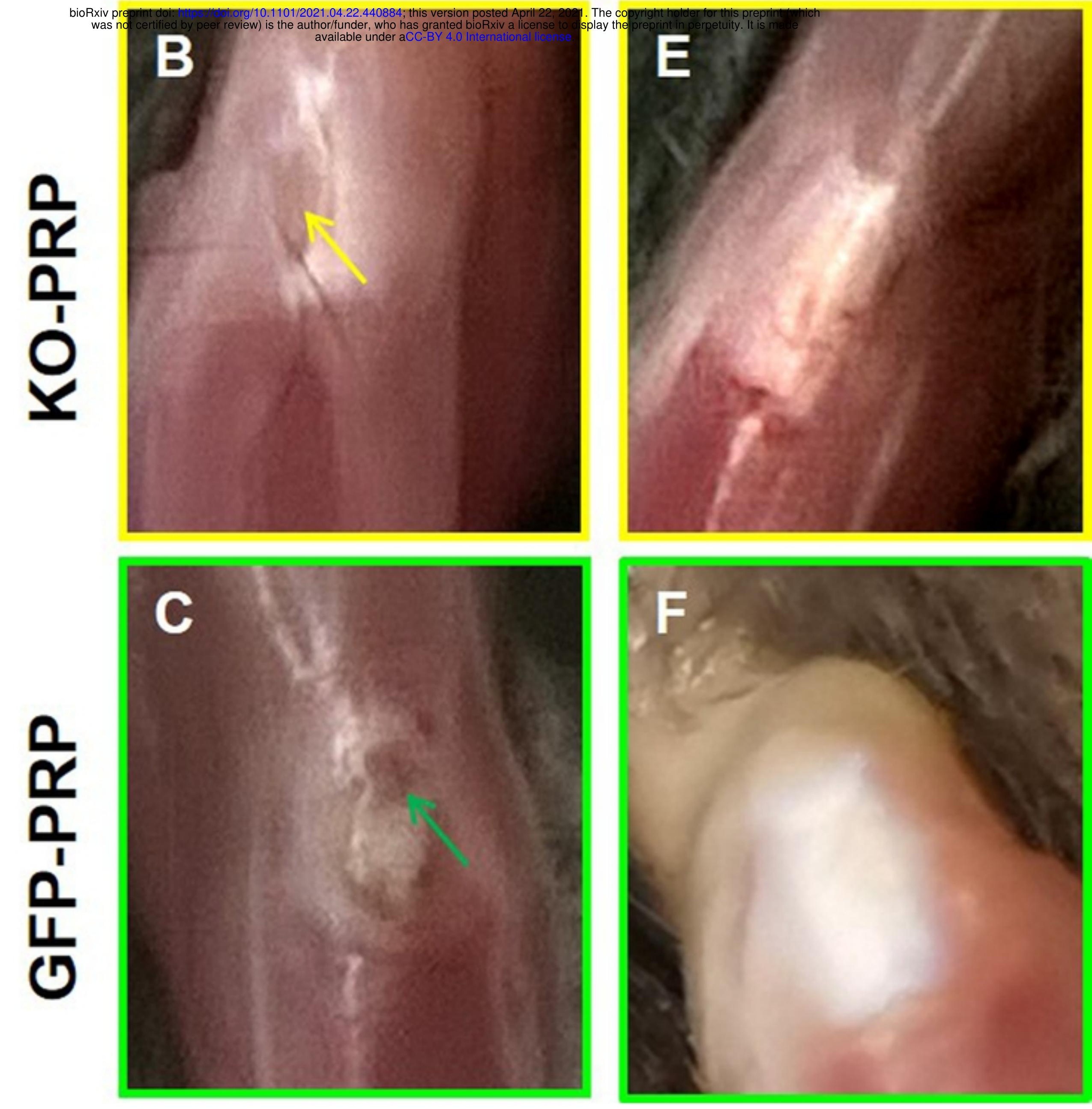

Fig2 
DAPI+HMGB1
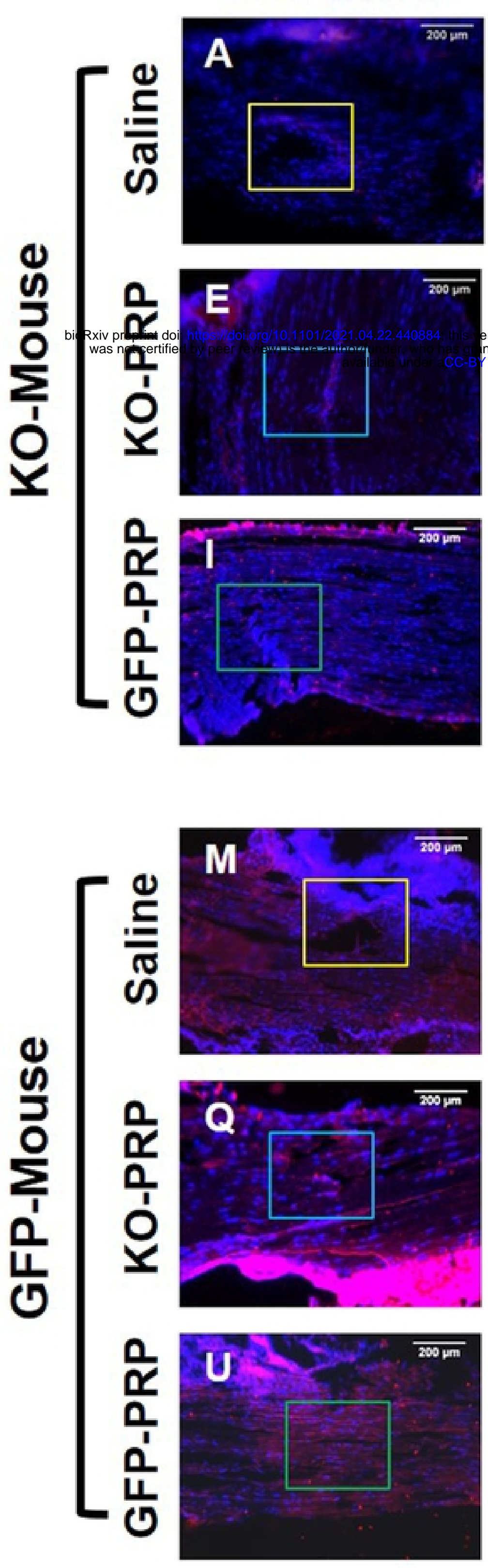
Enlarged

DAPI+HMGB1+GFP

Enlarged
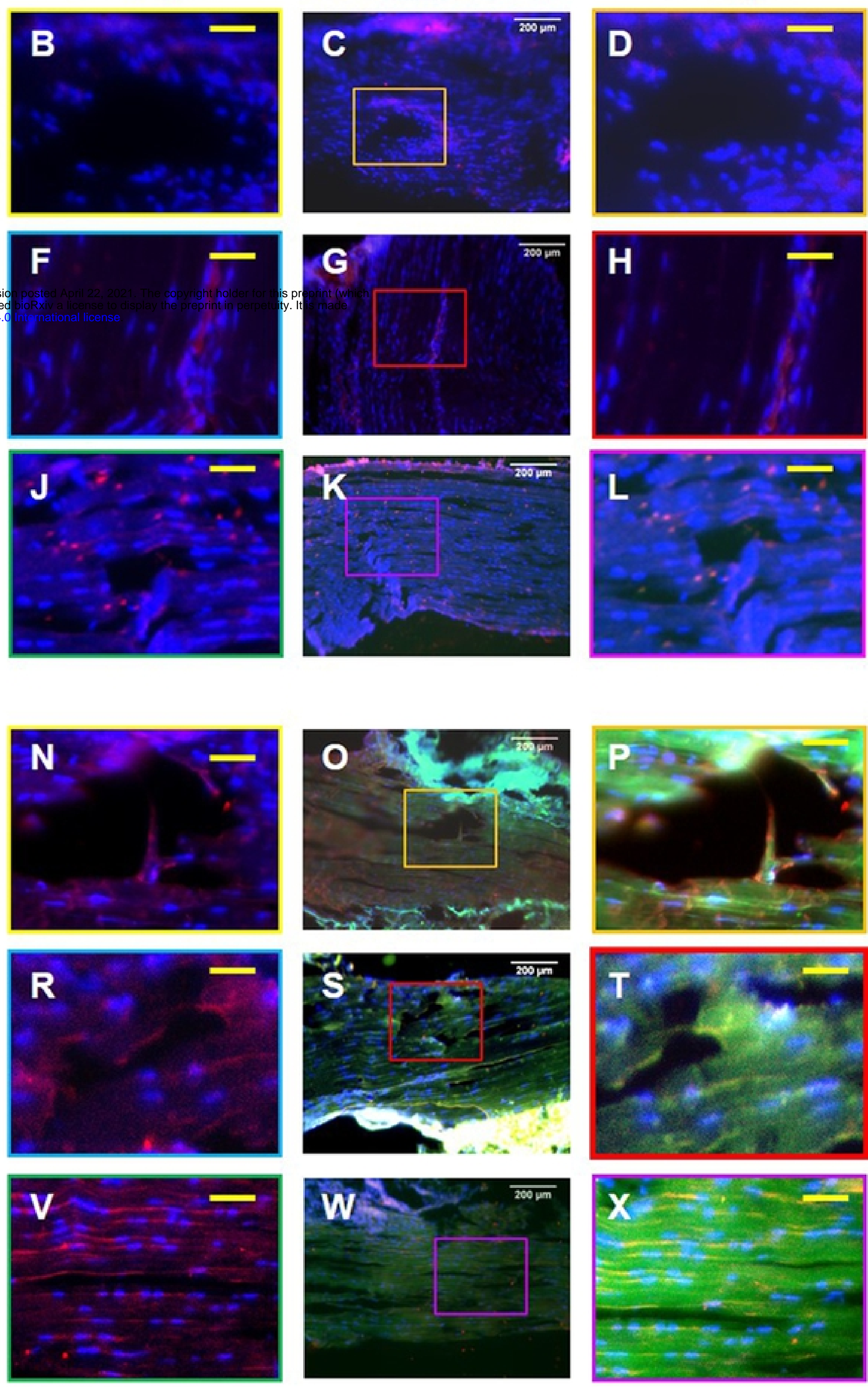

Fig4 


\section{Saline}

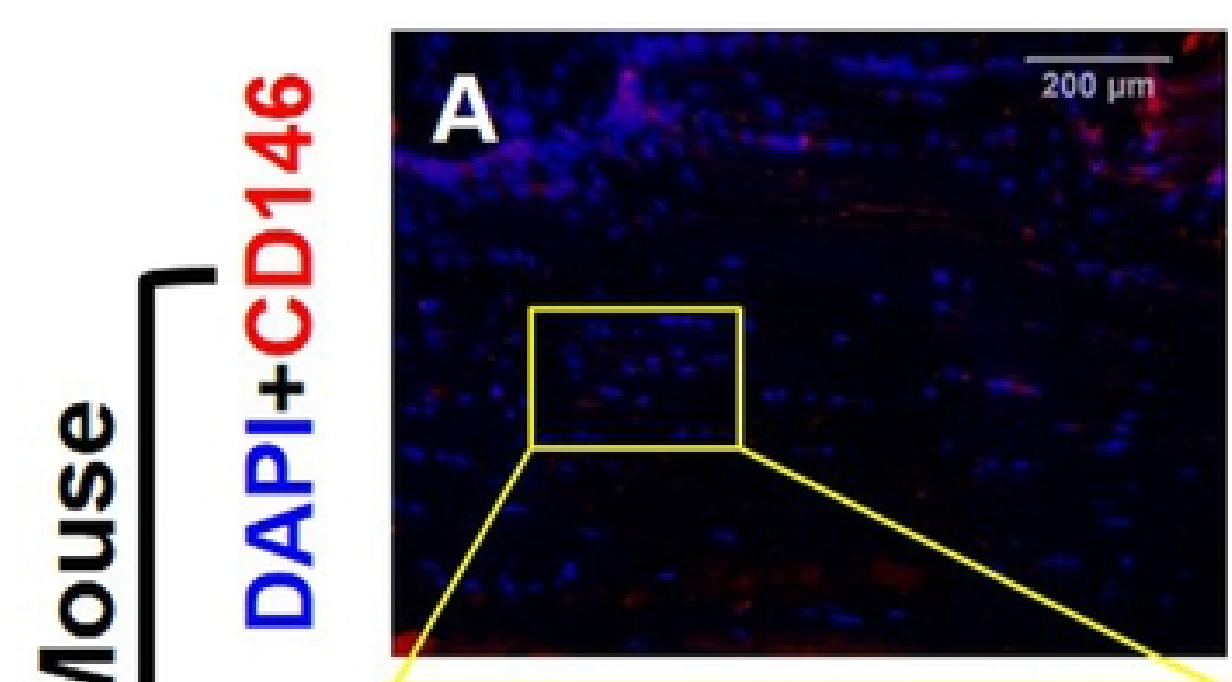

\section{B}
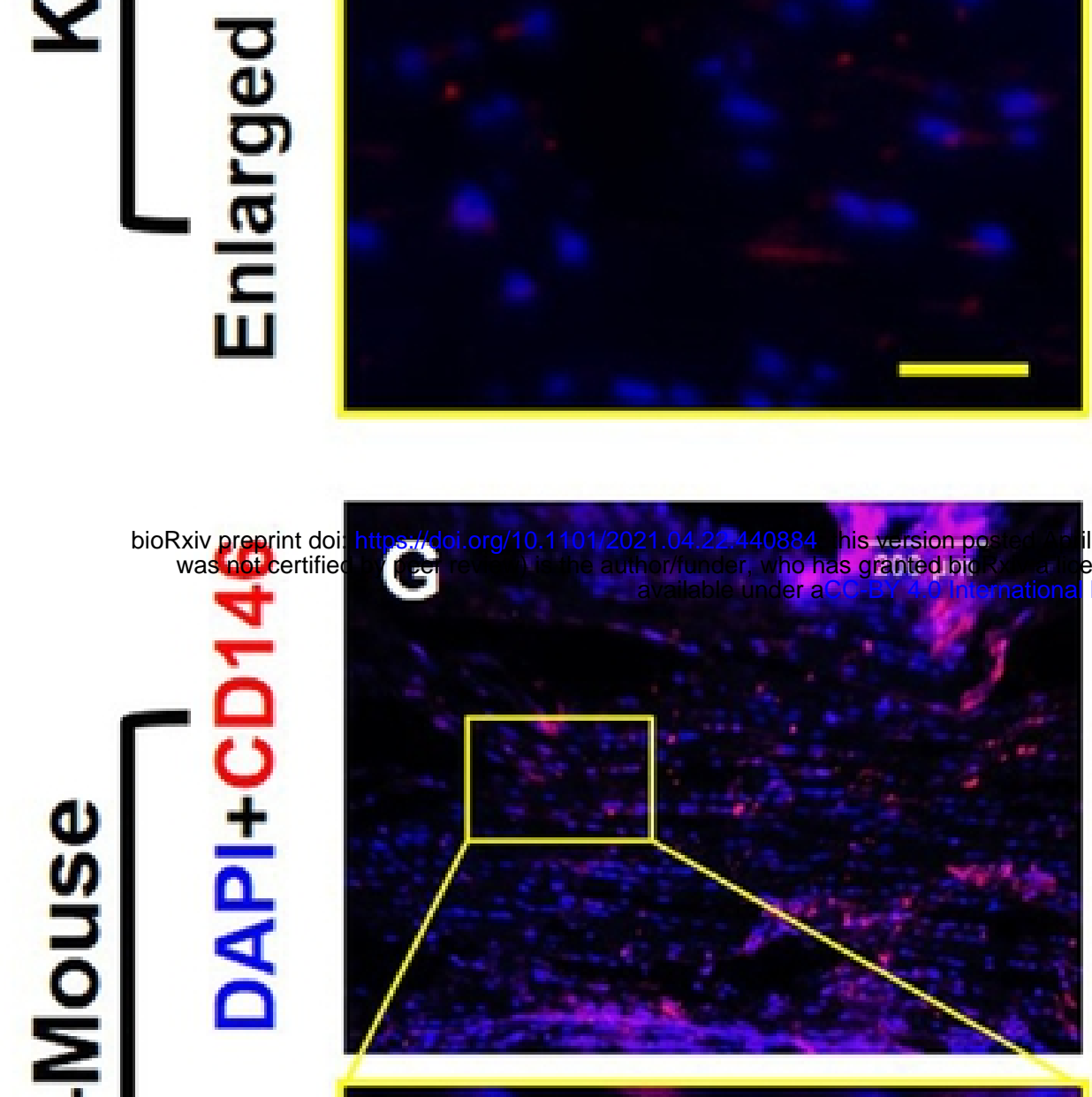

$$
\text { H }
$$

KO-PRP

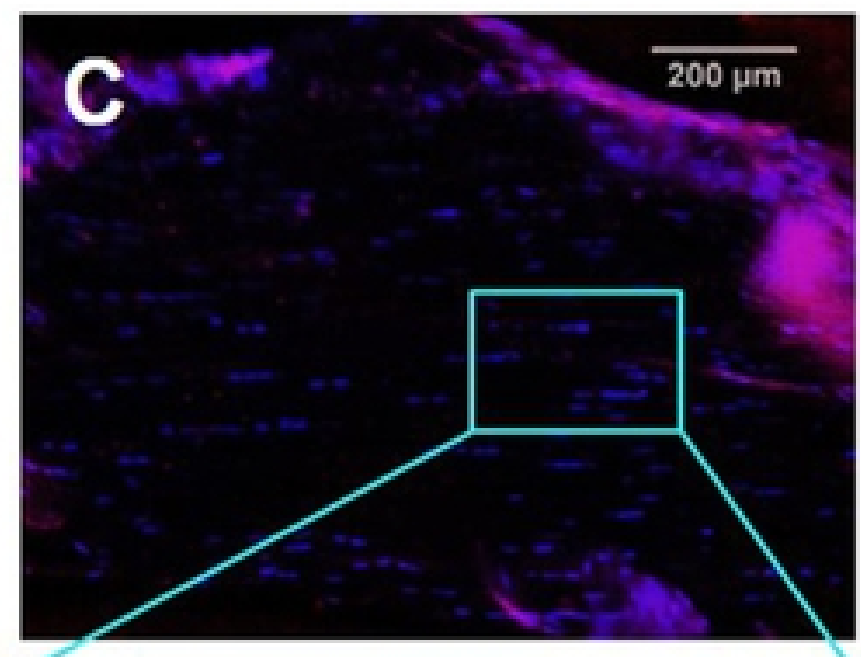

D

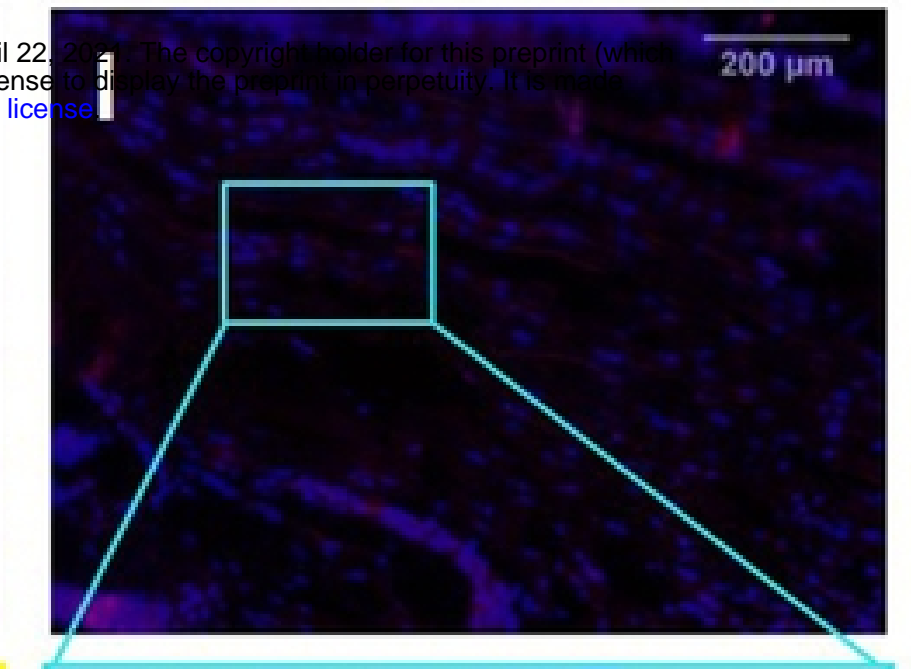

J
GFP-PRP

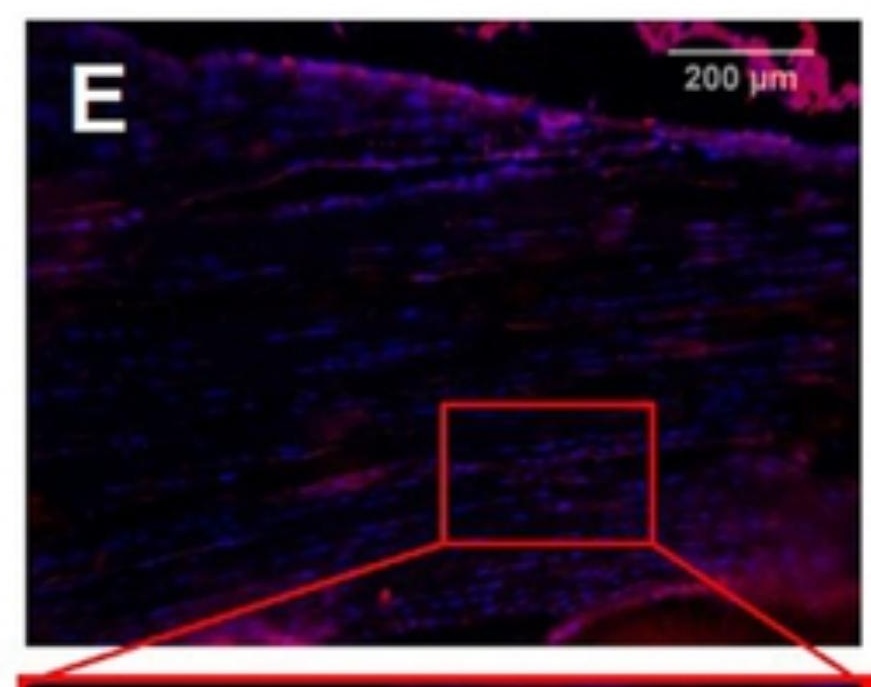

F

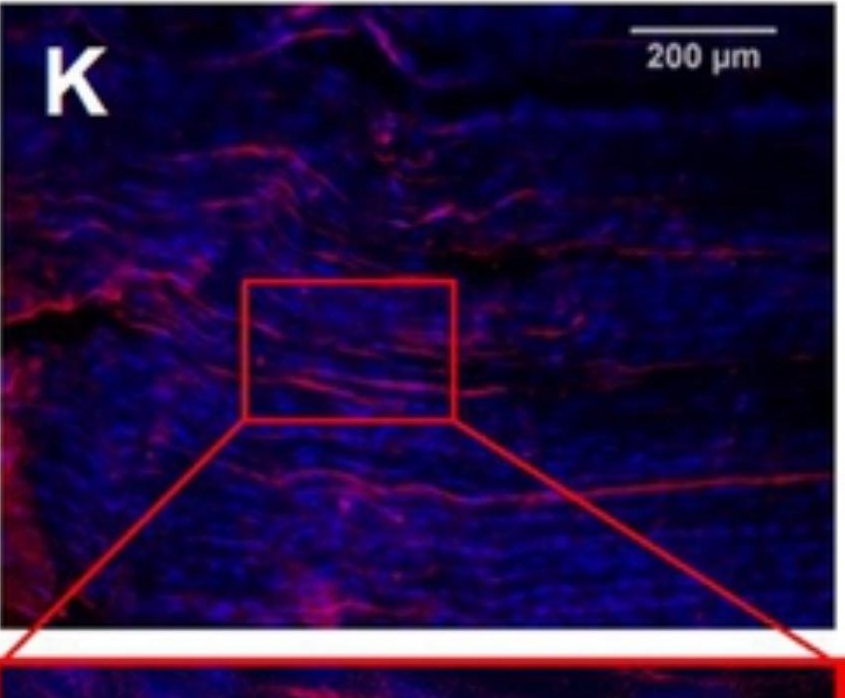

L

M

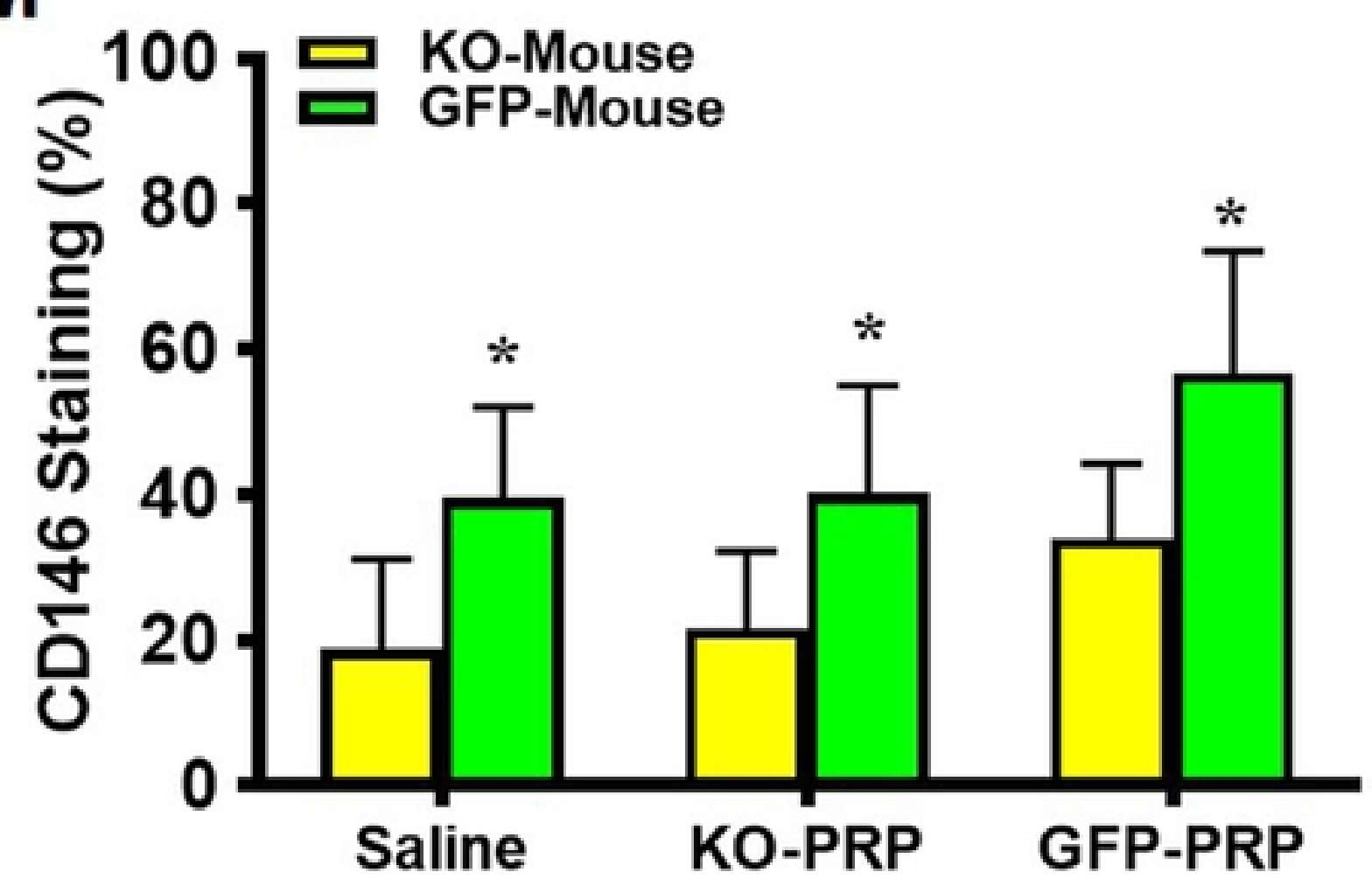

Fig6 


\section{Saline}

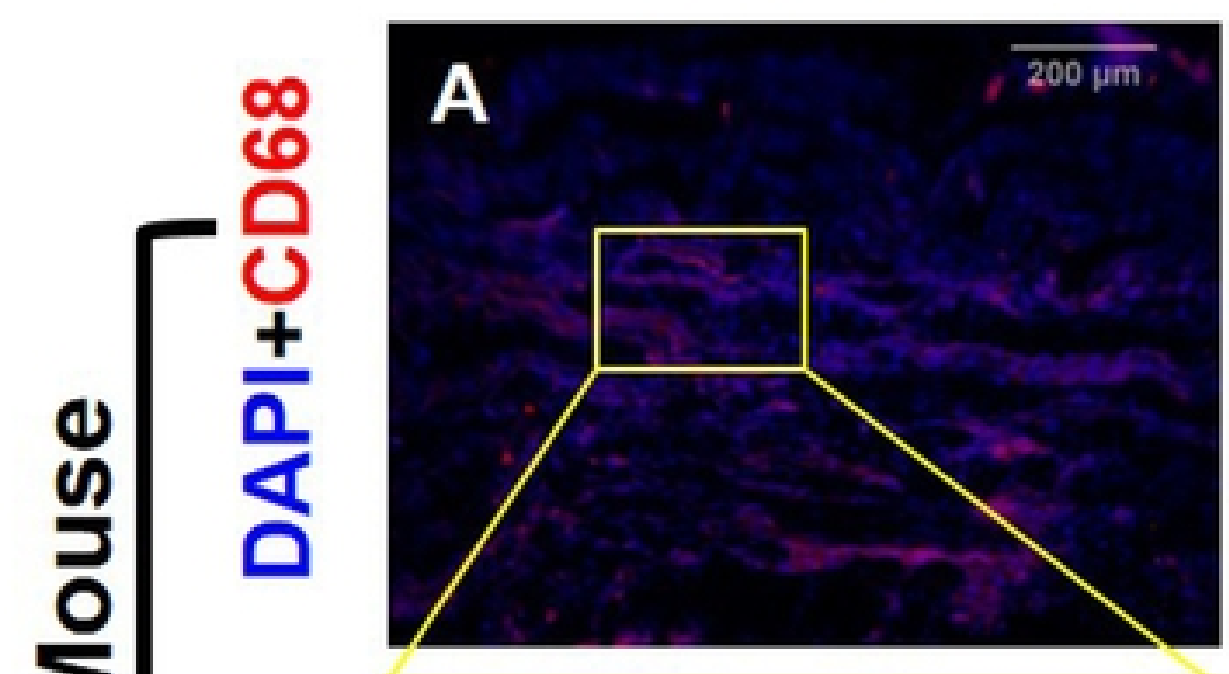

B

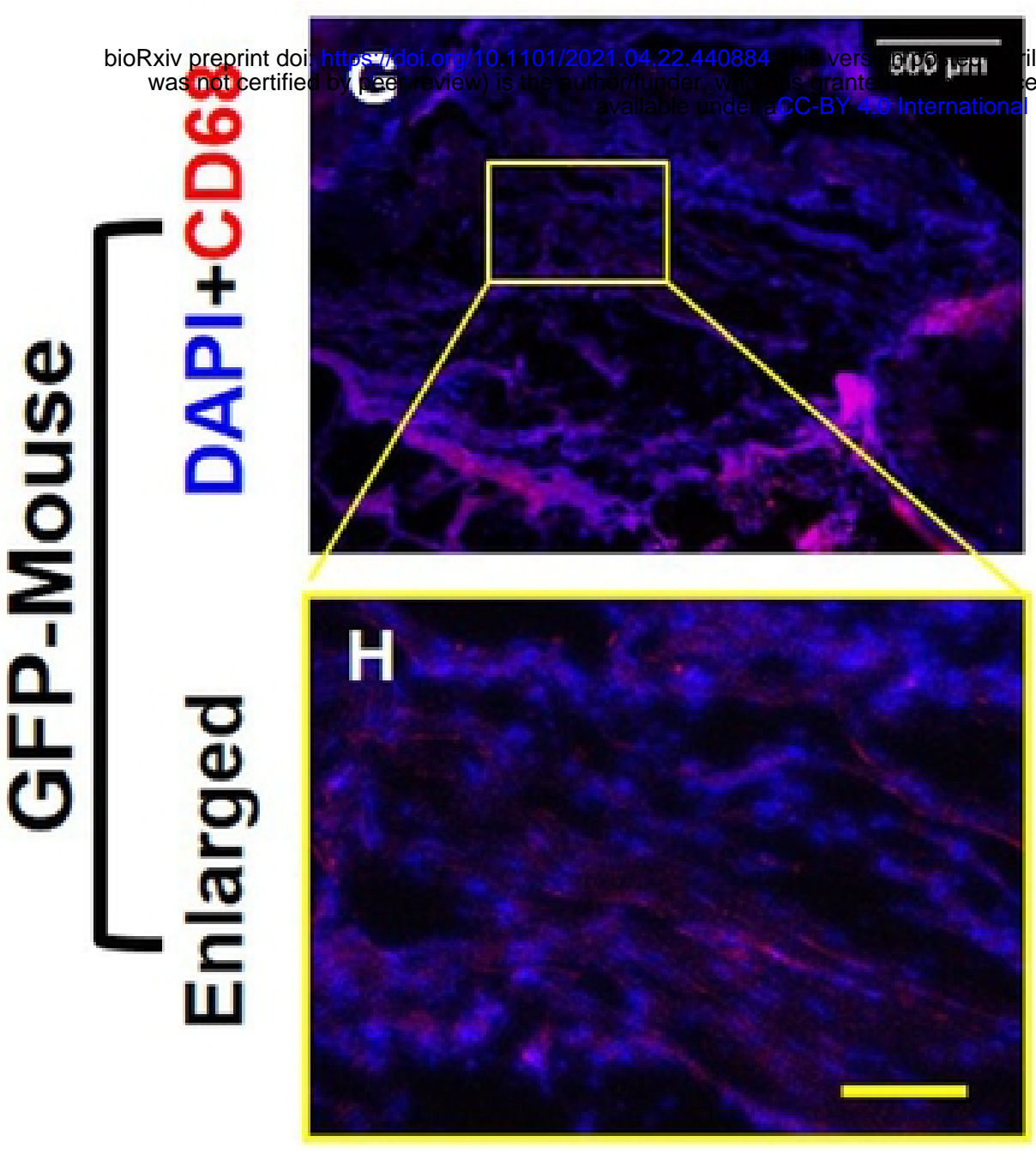

GFP-PRP

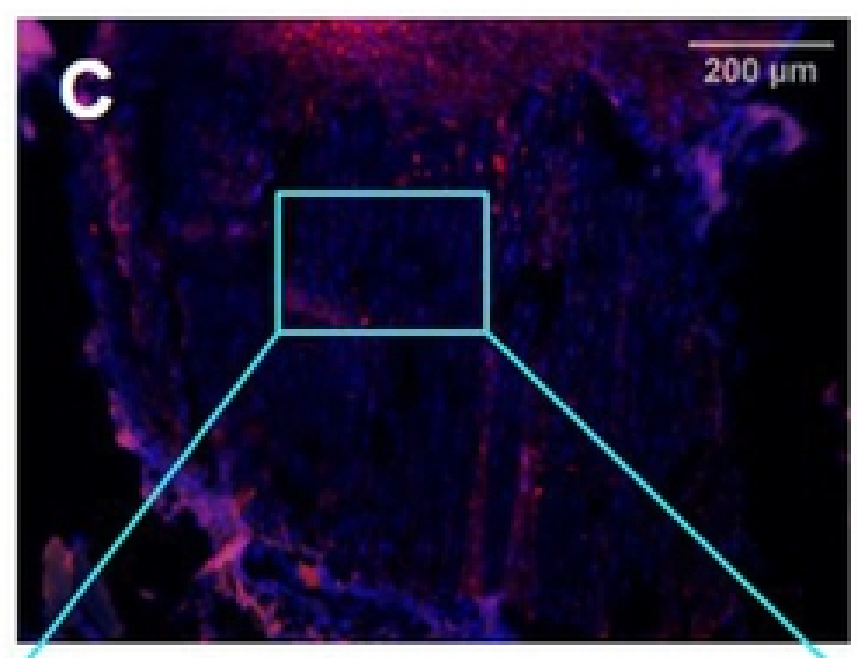

D

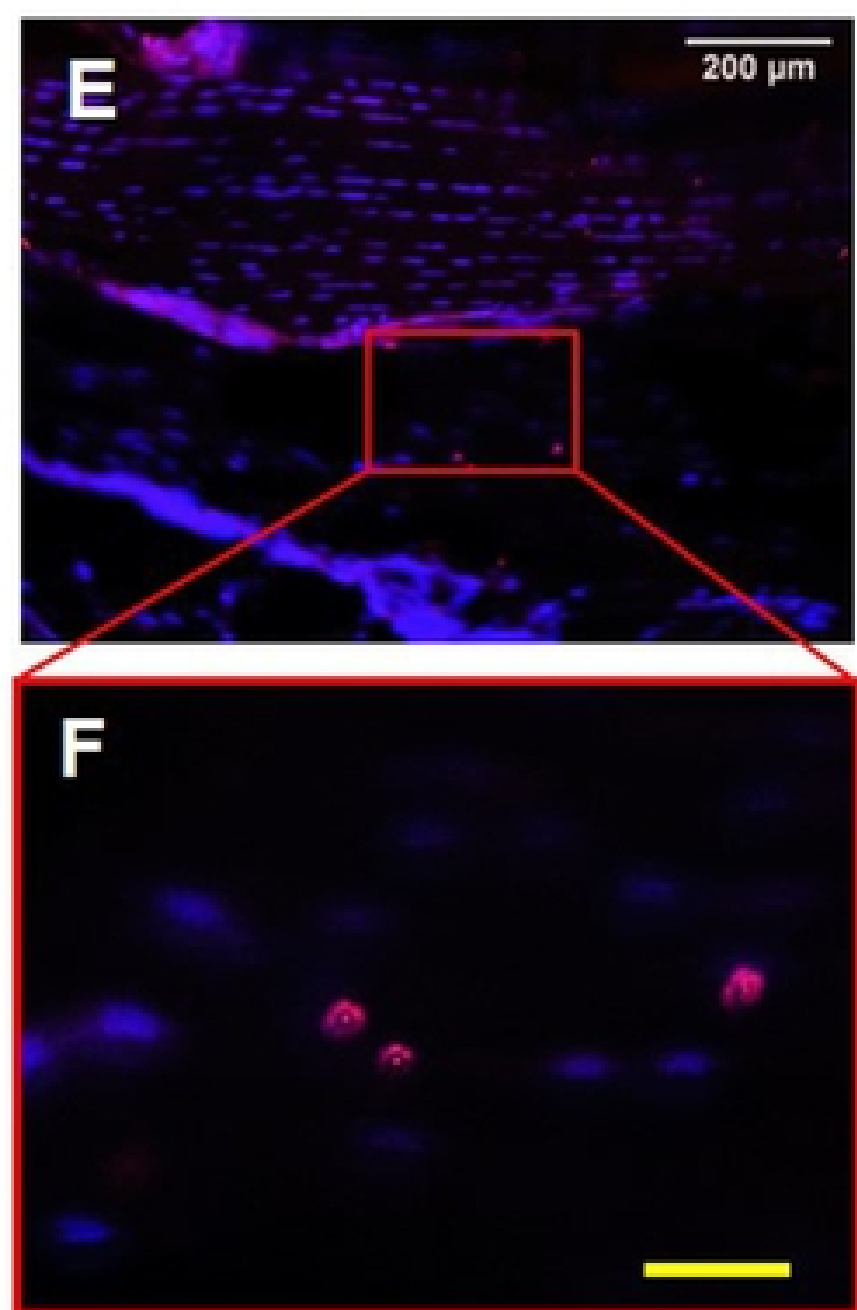

K

$\overline{200 \mu m}$

M

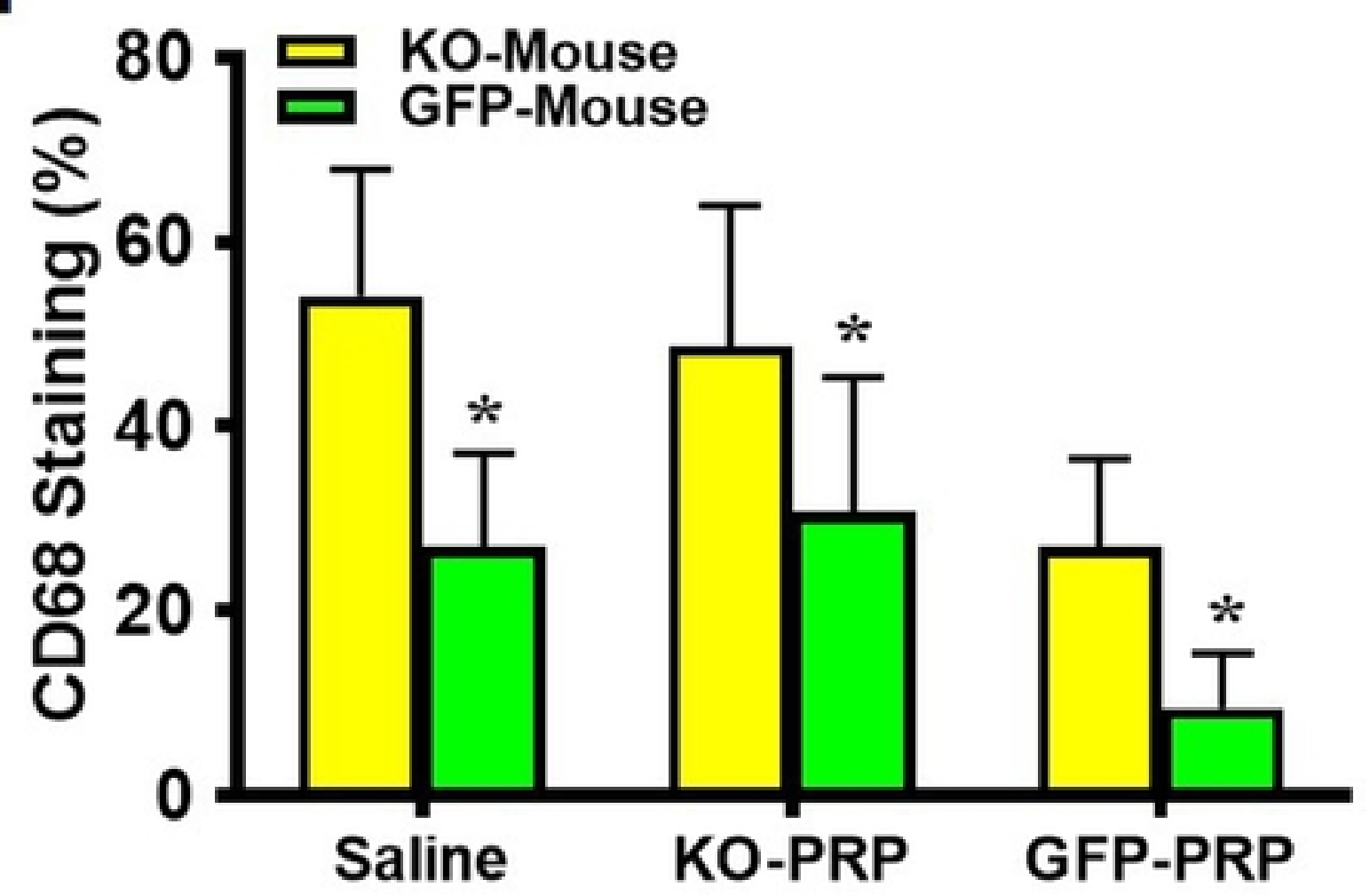

Fig5 


\section{Saline}
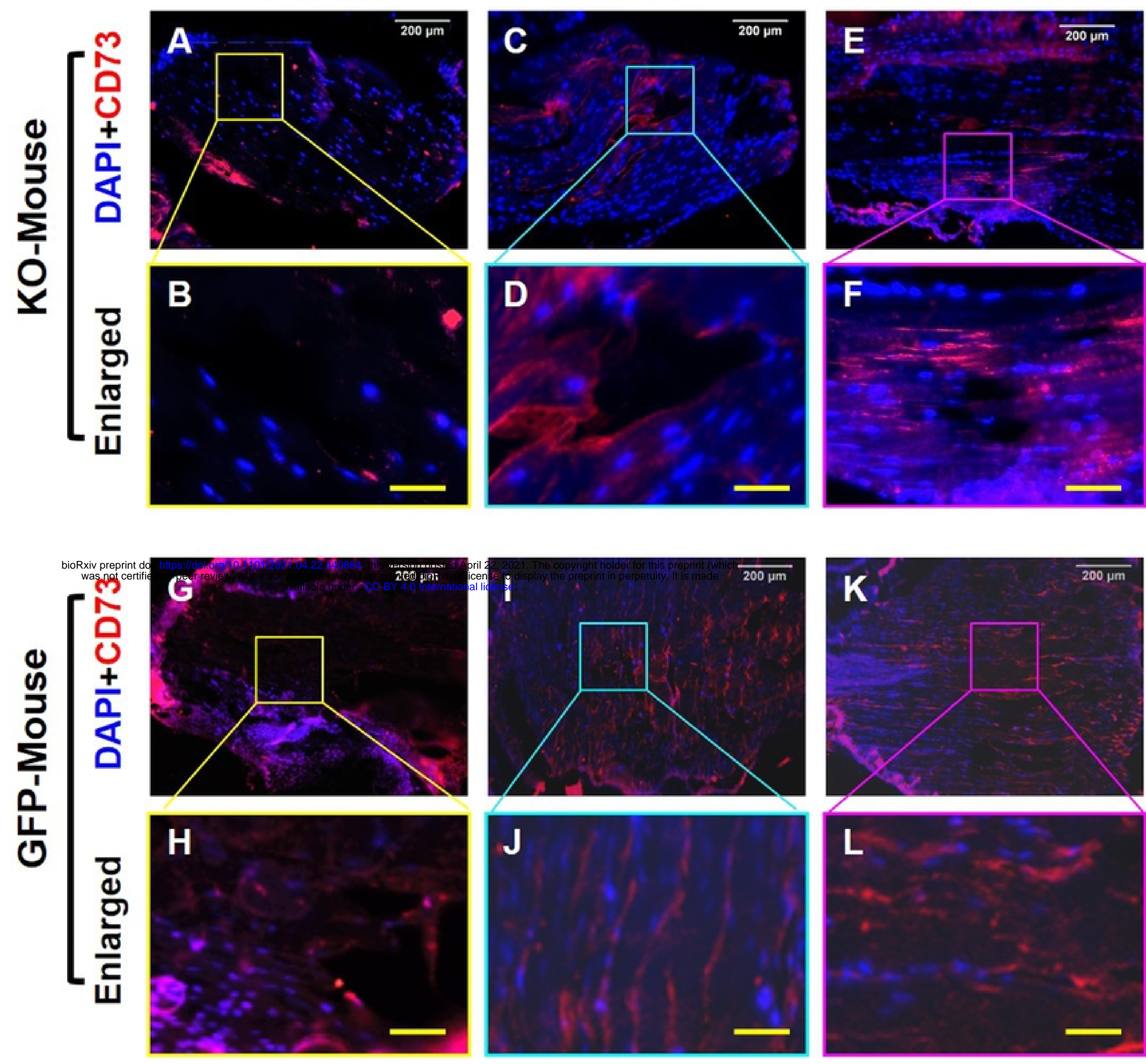

M

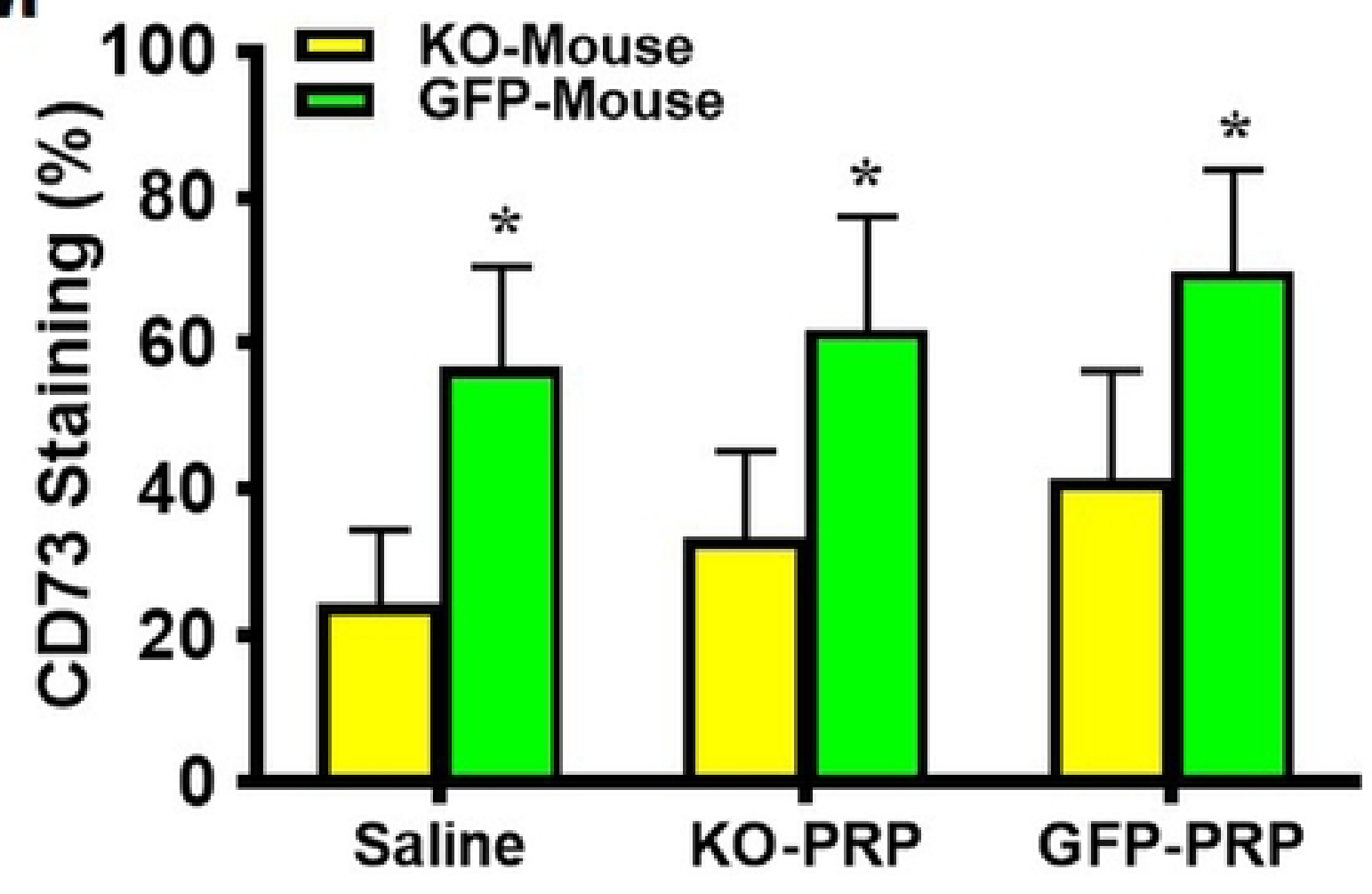

Fig7 
Saline
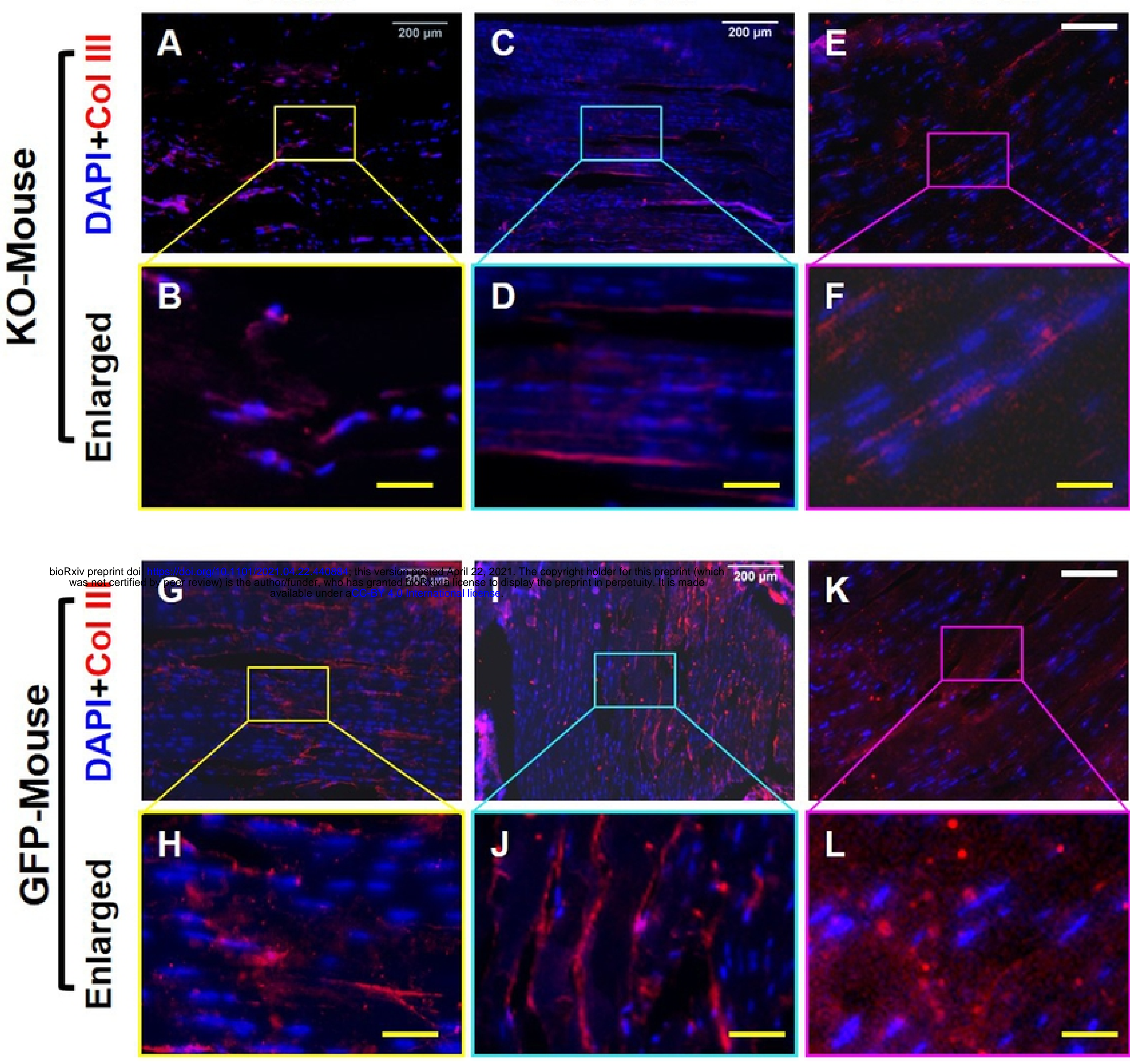

K
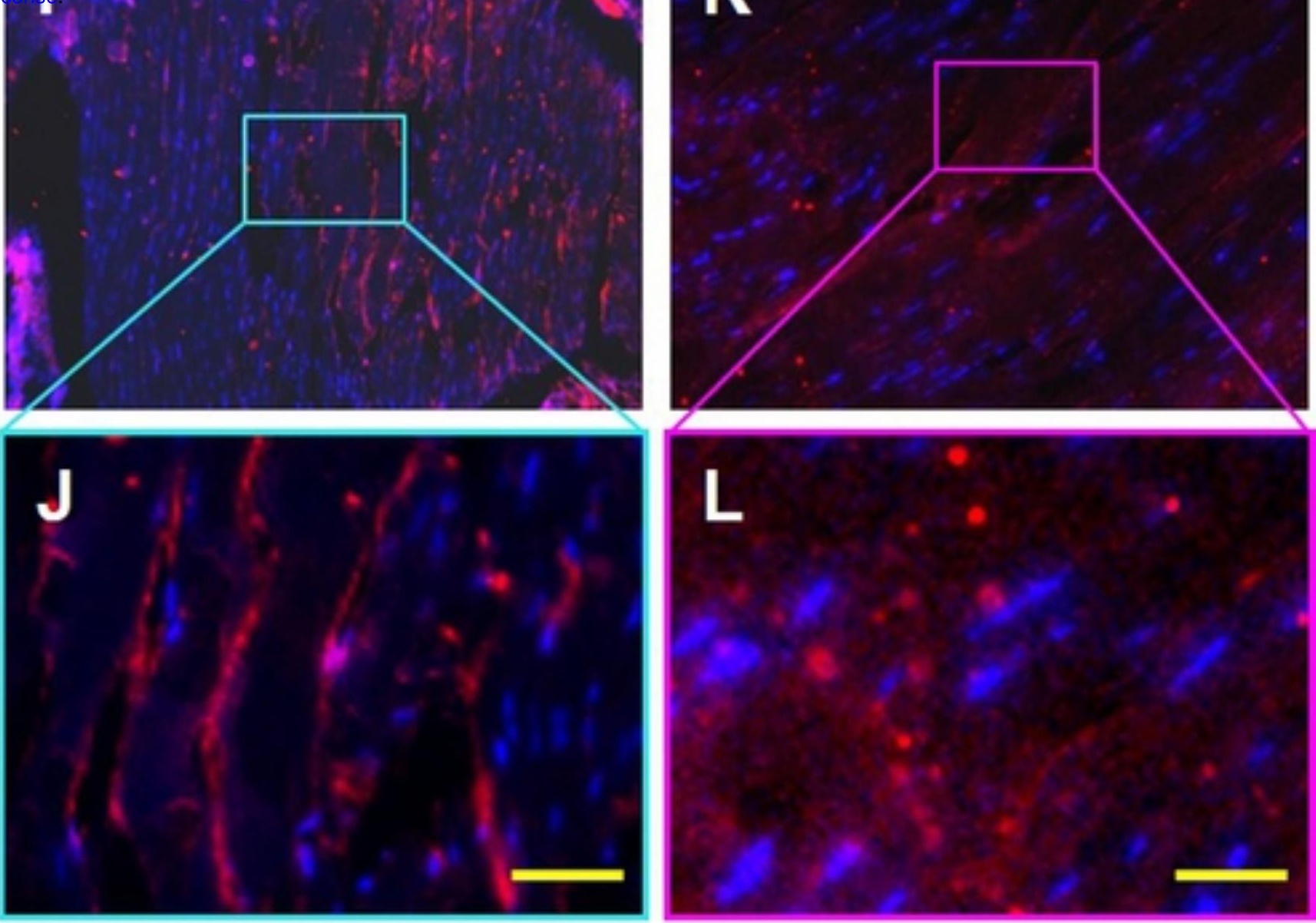

L

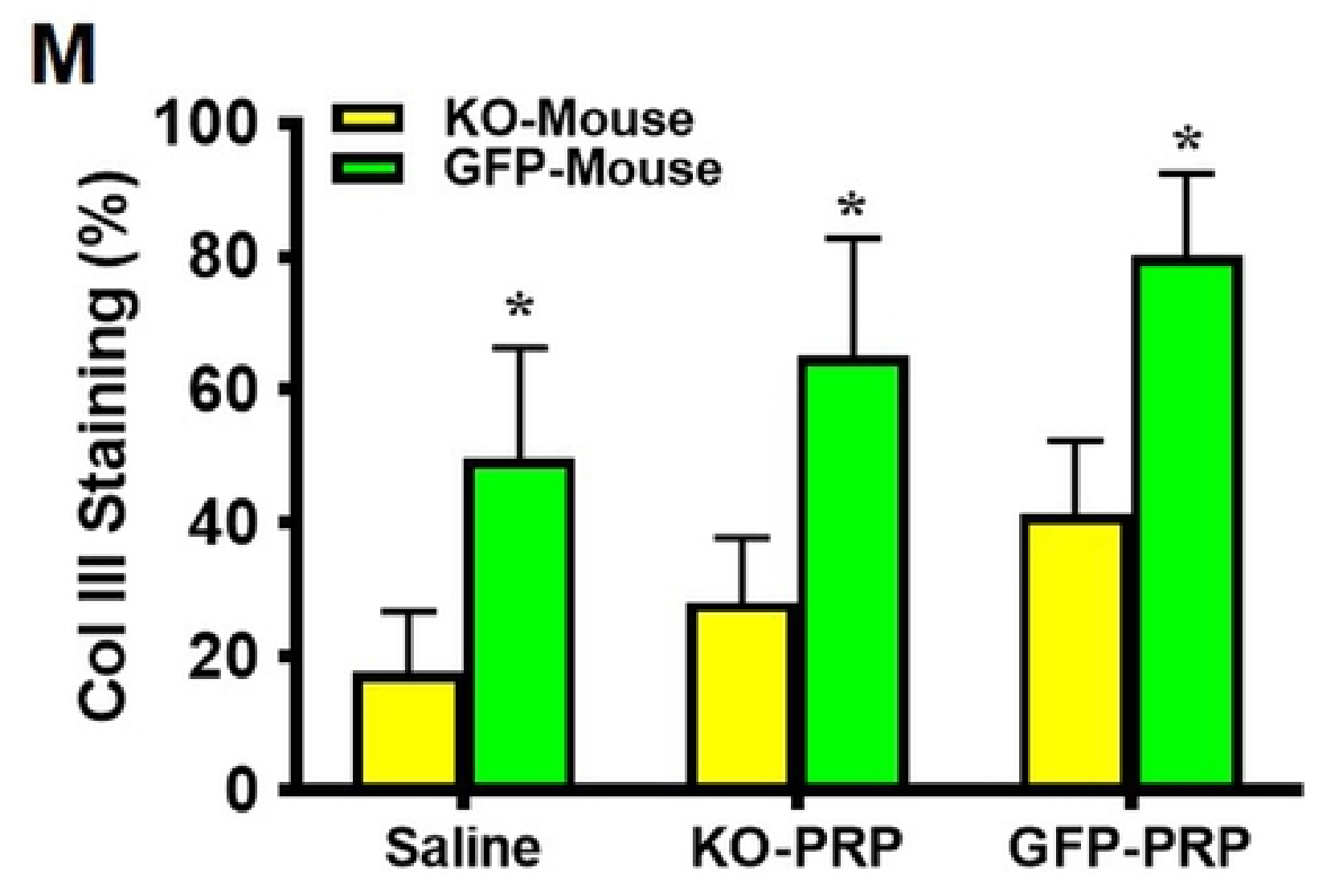

Fig8 


\section{KO-Mouse}

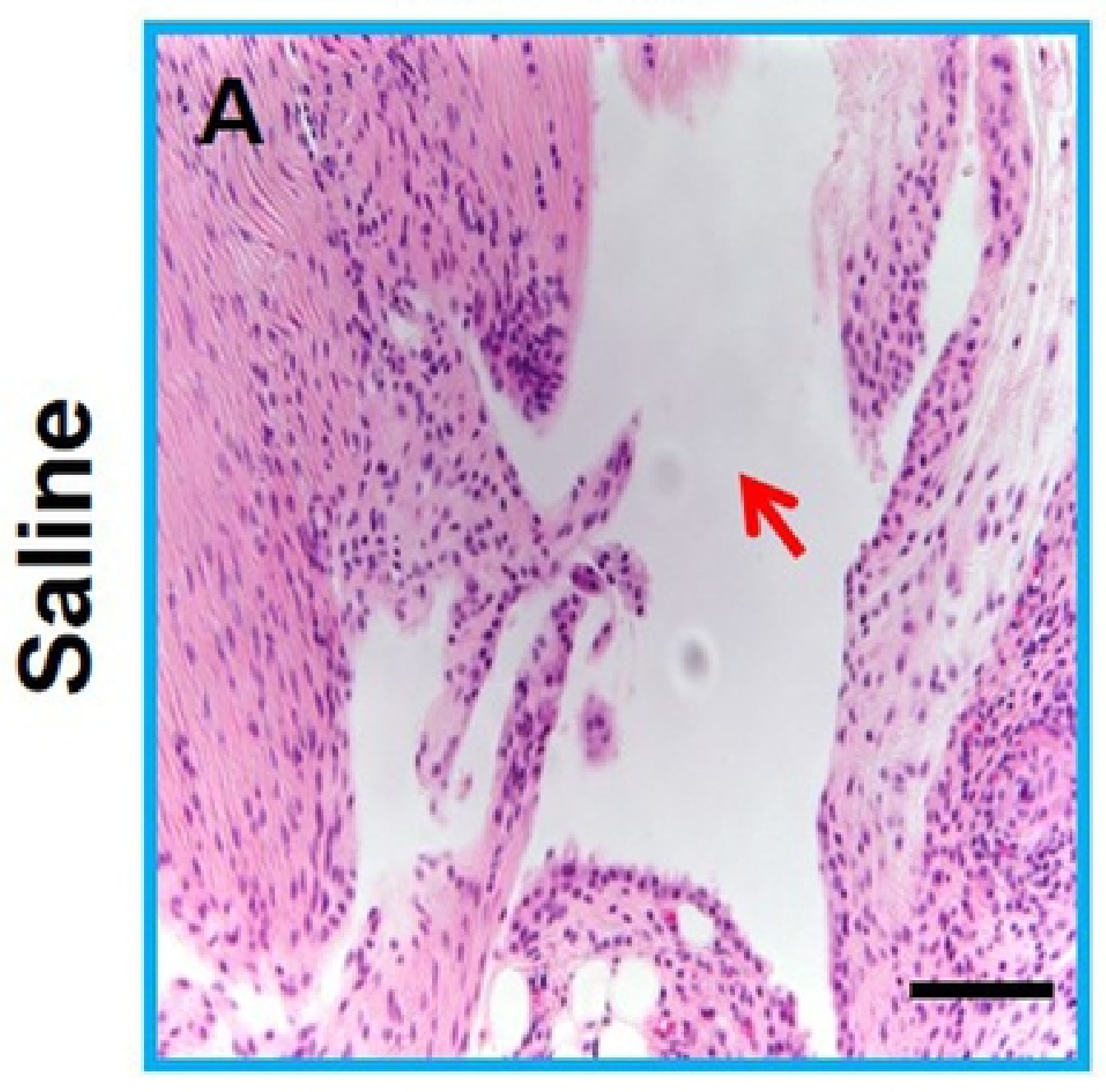

\section{GFP-Mouse}
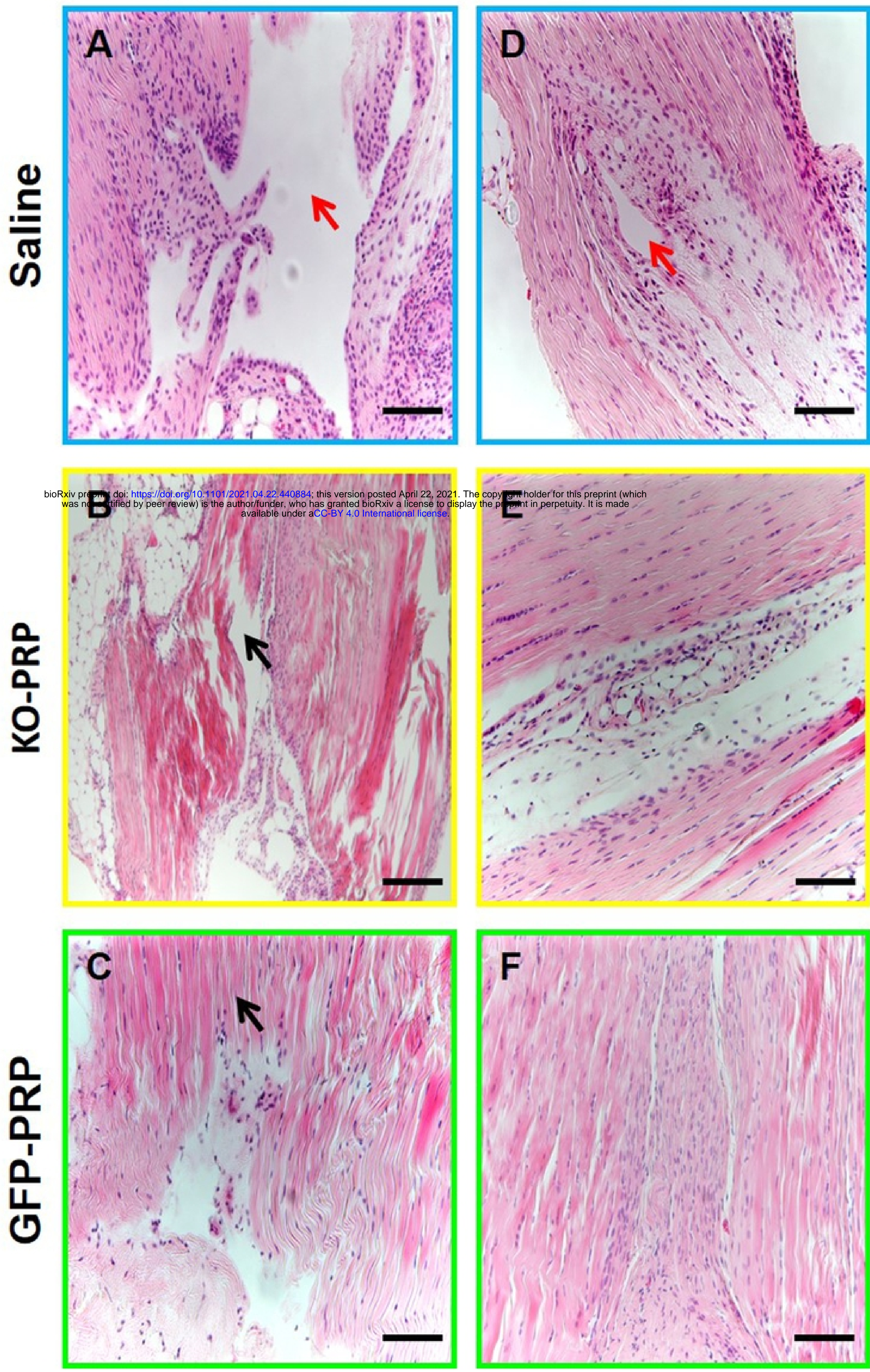
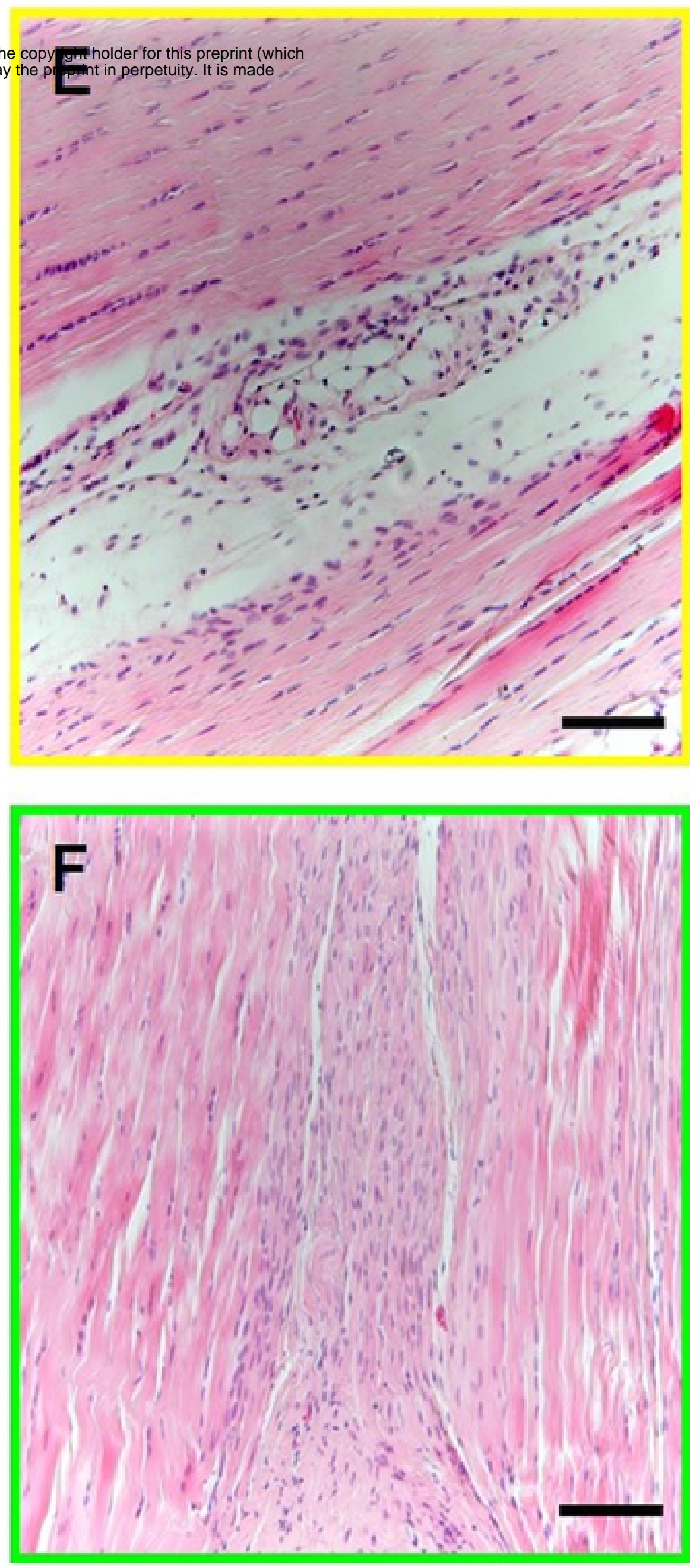

Fig3 